\title{
The Bearing Surfaces in Total Hip Arthroplasty - Options, Material Characteristics and Selection
}

\author{
Hamid Reza Seyyed Hosseinzadeh, Alireza Eajazi and Ali Sina Shahi \\ Shahid Beheshti Medical University Tehran
}

Iran

\section{Introduction}

During total hip arthroplasty, both the femoral and acetabular bearing surfaces are surgically replaced with metallic, polymeric, and/or ceramic components. Throughout the twentieth century, many different combinations of these materials have been proposed and examined as bearing surfaces for total hip arthroplasty. Metal-on-metal total hip replacements were first implanted by Wiles in the 1930s and later developed in the 1950s and 1960s by pioneering surgeons like McKee and Ring. In 1958, Charnley introduced a "low-friction arthroplasty" based on the principle of a metallic femoral component articulating against a polymeric acetabular component, and in 1970, Boutin developed the first ceramic-on-ceramic total hip replacement. Charnley's hard-on-soft bearing concept eventually dominated the hard-on-hard bearing alternatives. Today the most widely accepted bearing couple (i.e., combination of bearing materials for the hip joint) consists of a femoral head made from cobalt chromium molybdenum (cobalt chrome or CoCr) alloy articulating against a polymeric component fabricated from ultrahigh molecular weight polyethylene (UHMWPE). The use of the CoCr/UHMWPE bearing couple has proved consistent results in total hip arthroplasties around the world for the past four decades. (Older 2002)

In 1998, an estimated 700,000 UHMWPE hip components were implanted worldwide. Based on the report of major orthopaedic manufacturers, at most 200,000 metal-on-metal or ceramic-on-ceramic components have been implanted in patients worldwide between 1988 and 2000, corresponding to less than $10 \%$ of total hip replacements during the same time period. Therefore, the overwhelming majority (over 90\%) of total hip arthroplasties currently in service throughout the world includes an UHMWPE or a modified UHMWPE component and is based upon Charnley's original concept of hard-on-soft bearing. Despite the worldwide acceptance of total hip arthroplasty, wear of the UHMWPE component is a major obstacle limiting the longevity of these reconstructions. It is well established that particulate debris generated from the hard-on-soft articulating surfaces initiates a cascade of adverse tissue response leading to osteolysis and in certain cases loosening of the components. Extending the longevity of total joint replacements using alternative bearing technologies with improved wear behavior has been the subject of ongoing research in the orthopaedic community. Since the 1970s, researchers have attempted to improve the tribological characteristics of UHMWPE by modifying the polymer's structure, with the ultimate goal of improving the in vivo wear performance of hip bearings. In the 1970s, 
carbon fiber-reinforced UHMWPE with a potentially improved wear resistance was clinically introduced. In the 1980s, a high-pressure recrystallized form of UHMWPE was clinically introduced for its improved creep resistance. In the late 1990s, many researchers confirmed that crosslinking of UHMWPE can substantially improve the wear performance of the polyethylene in hip joint simulators. Based on these in vitro analyses, a number of radiation cross-linked materials have been clinically introduced in the late 1990s.

\section{Tribology of articulating surfaces}

According to the ASM International handbook, tribology is defined as the science and technology of interacting surfaces in relative motion and all practices related thereto. It includes the study of wear, friction, and lubrication. Total joint replacements have bearing surfaces that must transmit normal joint loads and motions. Low friction has been an important design objective for prosthetic joints for two reasons. First, if large shear forces due to friction are applied to the articulating surfaces, the risk of loosening may be increased. Second, the addition of frictional shear increases the stresses associated with surface damage due to contact, which can result in the release of wear debris to the surrounding tissue that also increases the risk of loosening.

\subsection{Friction}

Friction arises from the interaction between moving solids in contact and hinders (sliding friction) or prevents (static friction) motion. The friction force is proportional to the normal load applied to the surface and the coefficient of friction. The coefficient of friction is determined, in part, by the bearing surface material. There are two coefficients of friction, static and dynamic. It requires a greater force to initiate sliding than to maintain it, so the latter is generally considered to be $70 \%$ of the former. The contact area also determines the frictional force. The true contact area for many bearing surfaces is far less than the apparent contact area, somewhere in the region of $1 \%$.(Fig.1)

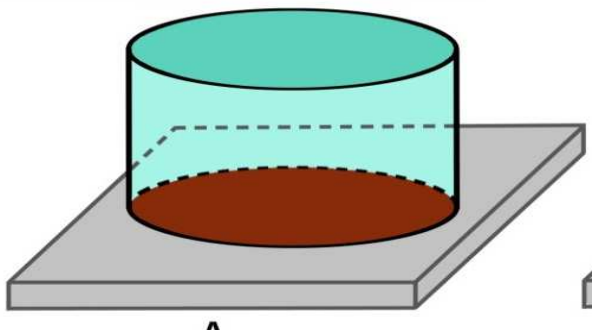

A

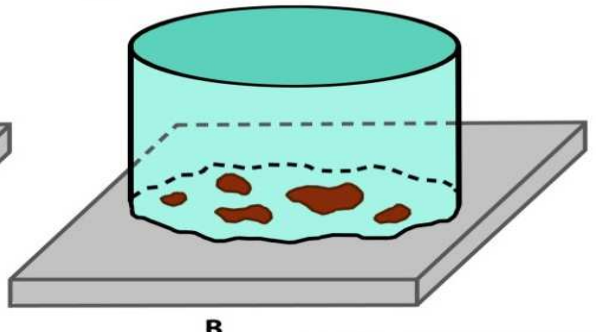

B

Fig. 1. Apparent (A) and Real (B) area of contact

This is due to the marked irregularity of the surfaces (asperities) at microscopic level. Only very small peaks from each surface contact each other.

The friction force is defined as the force acting tangentially to the interface resisting motion, when, under the action of an external force, one body moves or tends to move relative to another. The friction force $\mathrm{F}$ may be associated with sliding motion or with pure rolling motion of the bodies. 
The coefficient of friction $\mu$ is a dimensionless number, defined as the ratio $\mathrm{F} / \mathrm{N}$ between the friction force $\mathrm{F}$ and the normal force $\mathrm{N}$ acting to press the two bodies together. The kinetic coefficient of friction $\mu_{\mathrm{k}}$ is the coefficient of friction under conditions of macroscopic relative motion between the two bodies, while the static coefficient of friction $\mu_{\mathrm{s}}$ is the coefficient of friction corresponding to the maximum friction force that must be overcome to initiate macroscopic motion between the two bodies. $\mathrm{F}$ is approximately linearly proportional to $\mathrm{N}$ over quite large ranges of $\mathrm{N}$. The equation

$$
\mathrm{F}=\mu \mathrm{N}
$$

is sometimes called "Amontons Law". The value of $\mu$ can be expected to depend significantly on the precise composition, topography and history of the surfaces in contact, the environment to which they are exposed, and the precise details of the loading conditions. Although tables of coefficients of friction have been published, they should not be regarded as anything more than general indications of relative values under the specific conditions of measurement. The coefficient of friction usually lies in the range from 0 to 1 , although there is no fundamental reason why it need do so.

In order to initiate sliding, the bonds between the contact points need to be broken, thus explaining the higher force required to initiate movement with respect to maintaining it. The friction begins by cyclic elastic or plastic deformation of contact spots of the real contact area. Then it is transformed into elastic or plastic deformation energy within interlocking surface asperities and/or leads to crack initiation and propagation. This deformation may be responsible for the generation of particles. This (progressive) loss of particulate debris from the surface of a solid body due to mechanical action has been defined as wear. The adhesion of the surface atoms and molecules of body and counterbody contributes to friction. The probability of adhesion depends on mechanical properties and the tendency of atoms and molecules to react chemically. Both the deformation and adhesion contributions to friction can be distinctly lowered by means of surface modifications or coatings as well as by lubrication. At least $90 \%$ of the introduced energy is dissipated into heat, leading to an increase of the temperature within the contact zone. Depending on local normal forces and the relative velocity, the average as well as the flash temperatures may rise. Although the average temperature is primarily governed by the normal force, the flash temperature depends mostly on the relative velocity and lasts for only a few nanoseconds or milliseconds. The remaining $10 \%$ is dissipated by storing mechanical energy within lattice defects generated by cyclic elastic and plastic deformation, phase transformation or chemical reaction of body, counterbody, interfacial medium, and environment. Friction also results in the transfer of force from the articulating areas to the fixed interfaces.

\subsection{Lubrication}

Friction can be reduced by lubrication. The principal idea behind lubrication is to interpose a material between two contacting solids to minimize interaction between them. For example, wetting of the surfaces reduces adhesion. The extent of fluid film formation plays an important role in the wear process of artificial joints in vivo. The effectiveness of a lubricant film can be defined by the specific film thickness which is dependent on the viscosity of the lubricant, the relative velocity between body and counterbody, the pressure across the interface, and the roughness of the mating surfaces. 
Sommerfield number is the determiner of the thickness of the lubrication fluid and depends on a number of factors:

- Viscosity

- $\quad$ Sliding velocity (velocity at which the fluid is forced into gaps)

- $\quad$ Applied pressure

Sommerfield number $\propto$ Fluid viscosity $\times$ Sliding velocity/Applied pressure

The higher the value, the thicker the lubrication film.(Howcroft 2008)

The wettability of the materials also plays a part. This is essentially describes how hydrophobic or hydrophilic they are. The ceramics are the most wettable of the currently used bearings.

The lambda ratio $(\lambda)$ refers to the ratio of fluid-film thickness to the surfaces roughness. Lambda values greater than 3 imply that the fluid-film thickness is greater than the height of asperities on the articular surface and represent fluid-film lubrication. Lambda values between 1 and 3 represent mixed film lubrication, and values less than 1 represent boundary lubrication.

Lubrication between the bearing surfaces of hip implants and its effect on friction generated during articulation is commonly illustrated by a Stribeck diagram. (Fig.2)

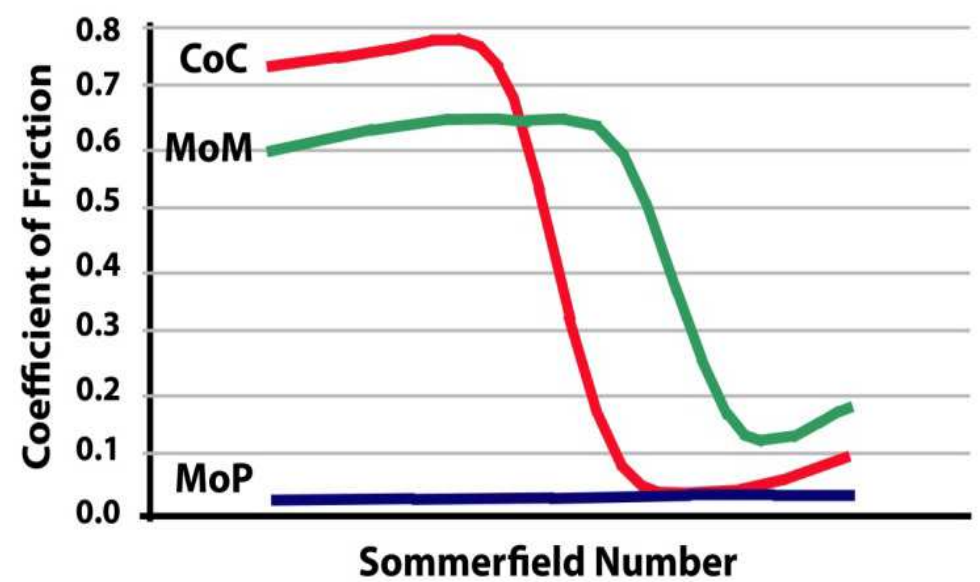

Fig. 2. The Stribeck diagram of different bearing combination materials

The Stribeck curve is traditionally depicted in three phases. When the thickness of the fluid film is less than or equal to the average surface roughness of the articulating surfaces, boundary lubrication (BL) is achieved. In this phase, the asperities of the articulating surfaces are in contact at all times. It is not ideal and is more likely to occur in rough bearing surfaces, or as a result of third body formation or protein deposition. It is improved with better manufacture tolerances of the bearing surfaces. The longer implants remain in situ the more likely they are to develop this type of lubrication. As the thickness of the fluid film increases, the articulating surfaces become separated from each other. There is a transition stage called mixed lubrication (ML), where there is a combination of 
fluid film and boundary lubrication. The coefficient of friction continues decreasing until full fluid film lubrication (FFL) is generated, where the articulating surfaces are separated by the lubricant. It is also called hydrodynamic lubrication and is divided into two groups depending on whether the two surfaces are conforming or not. Native joints are conforming (hydrodynamic lubrication) unlike artificial joints that deform elastically (elastohydrodynamic lubrication). Elastohydrodynamic (EHD) lubrication occurs when the pressure in the fluid film is sufficiently high to deform the asperities of the solid surfaces. Thus, even if the thickness of the fluid film is less than the heights of the asperities of body and counterbody, a total separation may be still achieved. Under realistic loads and in the presence of synovial fluid, metal-on-polyethylene hip joints articulate in the mixed film or boundary lubrication regime. Hard-on-hard bearings primarily work in the elastohydrodynamic and mixed film lubrication regime; however, with increasing femoral head size $(>28 \mathrm{~mm})$, a shift toward full fluid film (hydrodynamic) lubrication can be observed as well. (Smith 2001b; Dowson 2006)

\subsection{Wear and wear mechanisms}

Wear can be defined as damage to a solid surface, generally involving progressive loss of material, due to relative motion between that surface and a contacting substance or substances. Materials in contact are subjected to relative motion in many different applications. The creep and plastic deformation are not forms of wear per se because they do not produce wear debris but dimensional changes of the contacting surfaces. Also, corrosion is not directly related to wear because it can take place without any mechanical activation at all.(Buford 2004)

\subsubsection{Types of wear}

It is important, especially when describing wear, to distinguish clearly between the nature of the relative motion responsible for the wear and the physical mechanisms by which the material is removed or displaced in wear. The wear mechanisms in bearing surfaces are as follows:

\subsubsection{Adhesive wear}

The bonds that form between to surfaces need to be broken to allow movement. If the bonds are the weakest point then they will break. But sometimes one of the materials is weaker than the bonds so it breaks preferentially. Thus a layer of the weaker material lines the stronger material, changing the interface at which movement takes place. During mechanical action, these microjunctions are torn off, and fragments may become particles or be transferred from body to counterbody and vice versa, bringing about surface damage in the form of flakes and pitting. If the generated flakes and particles are bigger than the clearance of the bearing, they may act as abrasive particles or even block the joint.(Howcroft 2008)

\subsubsection{Abrasive wear}

When material is removed from a surface by hard asperities on the counterface or hard particles (third body) trapped between the two contact surfaces, abrasive wear occurs.(Howcroft 2008) (Fig.3) 


\section{Sliding Abrasion \\ (Two-Body)}

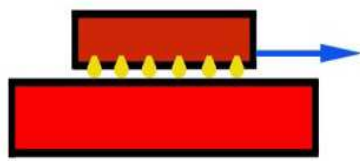

Rolling Abrasion

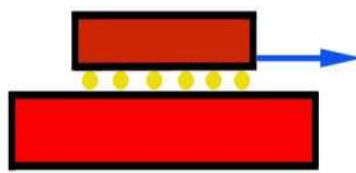

Scratching

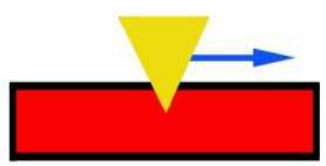

Fig. 3. Examples of abrasive wear

\subsubsection{Corrosive wear}

This type of wear occurs by the combination of mechanical wear and chemical reaction. (Howcroft 2008)

\subsubsection{Surface fatigue wear}

Fatigue acts during repeated sliding or rolling over the same wear track. The repeated loading and unloading can induce the initiation and propagation of microcracks parallel and orthogonal to the surfaces for mechanical or material-related reasons. As a result, shallow pits and filaments (delaminations) are generated.

\subsubsection{Wear mode}

The wear mode defines the general mechanical conditions under which the bearing is functioning when wear occurs. Wear modes are defined by two sets of criteria: first by the macroscopic structure of the tribosystem and the kinematic interaction of its elements and second by the combination of acting wear mechanisms. It should be noted that the wear mode is not a steady-state condition and can change from one form to another. For example, particulate debris generated by two-body abrasion may function as an interfacial medium and turn the problem into a particle-related (third-body) phenomenon. Depending on the circumstances, this may reduce or increase the wear rate. Mode 1 wear results from the motion of two primary bearing surfaces one against each other, as intended. Mode 2 refers to the condition of a primary bearing surface moving against a secondary surface, which is not intended. Usually, this mode of wear occurs after excessive wear in mode 1 . Mode 3 refers to the condition of the primary surfaces moving against each other, but with thirdbody particles interposed. In mode 3, the contaminant particles directly abrade one or both of the primary bearing surfaces. This is known as three-body abrasion or three-body wear. The primary bearing surfaces may be transiently or permanently roughened by this interaction, leading to a higher mode 1 wear rate. Mode 4 wear refers to two secondary (nonprimary) surfaces rubbing together. Examples of mode 4 wear include wear due to metal-cement or bone-cement interface motion or from relative motion of a porous coating, or other metallic surface, against bone; relative motion of the superior surface of a modular 
PE component against the metal support, so-called back-side wear; and fretting and corrosion of modular taper connections and extra-articular sources.(Jacobs 2006) (Fig.4)

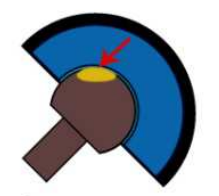

A
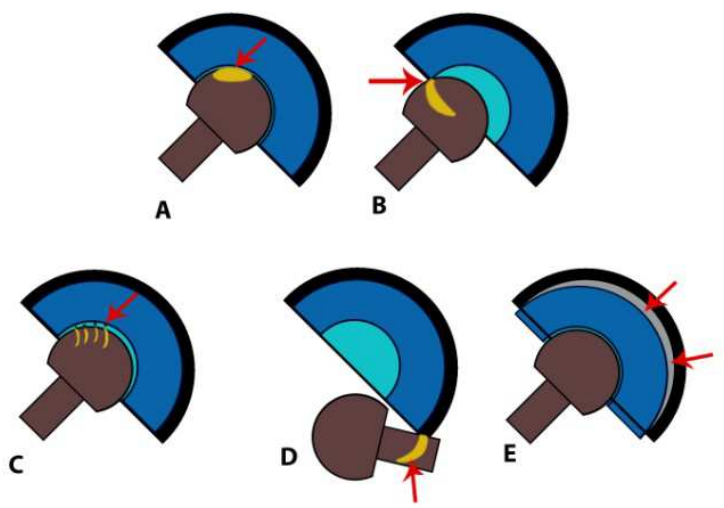

Fig. 4. The modes of wear for a total hip arthroplasty: A: Mode 1 or normal wear, B: Mode 2 or subluxation wear, C: Mode 3 or third body abrasive wear, D and E: Mode 4

Particles produced by mode 4 wear can migrate to the primary bearing surfaces, inducing third-body wear (mode 3). Wear particles are a function of the type of wear. A smooth, highly polished femoral head wearing against PE in the absence of third bodies generates very small wear particles with comparatively little variation in size and shape.

\subsection{Material properties}

Materials used in the manufacturing of femoral heads for metal-on-polyethylene (MOP) total hip replacement (THR) include the metal alloys, stainless steel, cobalt-chromium, and titanium alloy as well as ceramic materials, aluminum oxide, and zirconium oxide. Properties to consider when evaluating materials for bearings in THR include corrosion resistance, strength, ductility, hardness, and frictional characteristics. Frictional characteristics are a result of material properties such as wettability (related to surface energy), manufacturing variables such as surface finish, and operating conditions such as lubrication. The degree of resistance is proportional to the load. Because both chemical and mechanical interactions may occur, frictional forces depend on both the material composition and the roughness of the opposed surfaces. Lubricating conditions can change the nature of the interface between the moving surfaces and decrease friction. As described earlier, the coefficients of friction depend upon the nature and amount of lubricant present, as well as the speed of relative motion and the applied load.

\subsection{Surface roughness}

A thorough surface roughness evaluation should include a visual comparison of the actual tracings and representative photomicrographs (scanning electron microscope) of the surface. The surface roughness of currently available femoral heads ranges from an average height $\left(\mathrm{R}_{\mathrm{a}}\right.$ ) of less than $0.03 \mu \mathrm{m}$ to about $0.10 \mu \mathrm{m}$ and maximum height ranging from less than 0.10 to about $0.40 \mu \mathrm{m}$. The surface roughness of a femoral bearing can change over time in vivo. In the presence of hard third bodies, as can occur in vivo, surface abrasions (scratches) result 
in an increased surface roughness, and the wear rate of polyethylene (PE) can increase. Conversely, in "clean" operating conditions with little or no hard third bodies, motion against PE may result in polishing of the metal surface and a lower surface roughness. This suggests that differences in the initial surface roughness of a femoral head may not be as important in the long run as the in vivo operating conditions. The susceptibility to scratching is a function of the hardness of the material. The decreased hardness of titanium alloy results in decreased abrasion resistance. Although the initial surface roughness of a titanium alloy femoral head may be equivalent to that of other bearing materials, there is greater potential for surface roughness to increase in vivo. In an environment with few or no hard third bodies, the wear performance of titanium alloy against PE can be comparable to the other metals, but the performance of titanium alloy against PE is affected to a greater degree by the presence of hard third bodies. The abrasion resistance of cobalt-chromium alloy is considered superior to that of titanium alloy and stainless steel.(Fisher 2004)

The abrasive particles are mainly released from modular interfaces, metal backing, and/or porous coating. Increased femoral head surface roughness may dramatically accelerate twobody abrasive wear of PE. Experimental studies indicate that a threefold increase in femoral roughness can cause at least a tenfold increase in the wear rate of PE. Specific increases in wear rate are dependent on the nature of the damage to the femoral head. Ceramics are harder and therefore more resistant to damage by third-body particles than metal counterfaces. For this reason, the increased hardness of ceramic materials is considered advantageous. The pattern of damage by a hard third body in metals and ceramics differs.(Raimondi 2008) (Fig.5)
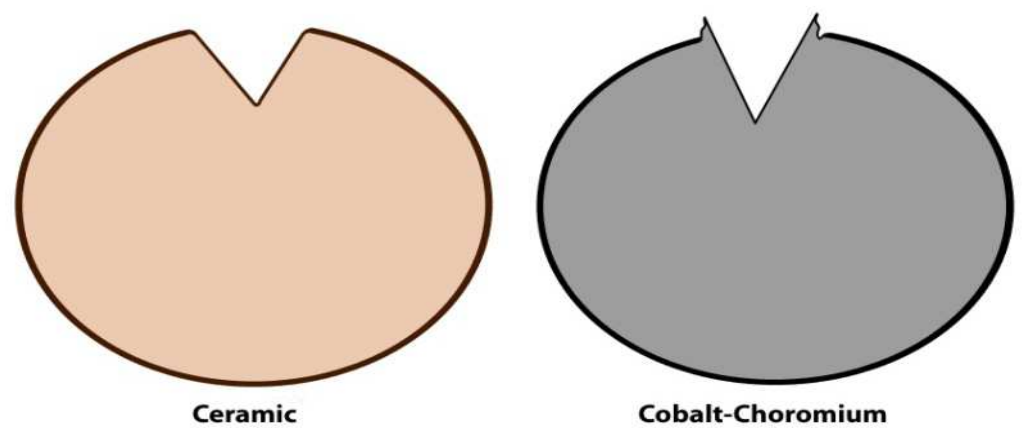

Fig. 5. Scratch profiles of metal and ceramic femoral heads

\subsection{Frictional torque}

The frictional torque of the Charnley prosthesis with a load of $890 \mathrm{~N}$ has been reported between 0.4 to $1.2 \mathrm{Nm}$. Under similar conditions, the frictional torque of a $28-\mathrm{mm}$ prosthesis, a $43-\mathrm{mm}$ prosthesis, and a 51-mm prosthesis averaged $1.3,2.7$, and $3.2 \mathrm{Nm}$, respectively. These values are 20 to 100 times smaller than the reported static torques to failure for cemented acetabular components. The coefficient of friction for the MOM bearing of the McKee-Farrar hip is roughly two to three times greater than that for the Charnley. The larger diameter of the McKee-Farrar (about $40 \mathrm{~mm}$ ) amplifies this difference, and the result is a frictional torque that is up to 10 times greater than that in the Charnley. This value is still an order of magnitude less than the static torque-to-failure of an acutely implanted 
cemented acetabular component and lower than that reported for surface replacement components. Contrary to theoretical considerations, frictional torque has not been demonstrated to be important in the initiation of aseptic loosening of either femoral or acetabular components. Accumulating evidence indicates that PE wear particles have a greater effect on the durability of implant fixation than frictional torque. From this perspective, the success of the Charnley low-friction arthroplasty is primarily a function of the low volumetric wear of the 22-mm bearing, not low frictional torque. Higher bearing surface friction and frictional torques can be tolerated if the release of wear particles to periprosthetic tissues is sufficiently low. Within the range of frictional torques generated by implants used to date, wear is a more important factor in survivorship than frictional torque. Large-diameter bearings can be successful if the wear rate is low. This is an important consideration as alternatives to $\mathrm{PE}$ bearings are being investigated.

\subsection{Stresses caused by contact}

When contact occurs between metal and polyethylene components, both surfaces deform, but the deformation of the metal component is negligible and the metal component behaves like a rigid indenter. Thus, when an artificial joint is loaded, the polyethylene is squeezed between the rigid metal component and the supporting material (bone, cement, or metal backing), and in the region of contact, the articulating surface of the polyethylene is forced to conform to the shape of the metal surface. The resulting deformation causes compressive, tensile, and shear stresses in the polyethylene. The magnitude of the stress depends on the magnitude of the joint load. These loads are large (3-5 times body weight) and in combination with the relative motion of the articulating surfaces, cause damage that increases with time of implantation (number of cycles of loading) and patient weight (magnitude). This provides strong evidence that surface damage in total joint replacements is the result of fatigue processes. The stresses associated with damage to the articulating surfaces occur both at the surface and within the polyethylene component. Two types of stresses can be applied to the surface: normal compressive stresses (contact stresses), and tangential shear stresses due to friction. Metal and polyethylene were originally chosen for bearing surface materials to produce low-friction total joint replacements. The stresses acting on the surface produce normal and shear stresses within the polyethylene. At the surface, the largest compressive stresses are the contact stresses that act perpendicular to the surface. They decrease nonlinearly with depth through the thickness of the polyethylene. Joint contact also produces compressive and tensile stresses within the polyethylene component that act tangent to the articulating surface. Tangential compressive stresses occur because the polyethylene under the center of the contact area expands radially as the component is compressed. This expansion is resisted by the surrounding material, and tangential compressive stresses are produced. Tangential tensile stresses near the articulating surface occur because the surface must stretch as the polyethylene conforms to the shape of the metal component when the joint is loaded. The stretching occurs near the edge of the contact area. The resulting tensile stresses are largest at the surface of the component. Surface damage is most likely due to combinations of stress components. The maximum shear stress occurs at the surface for conforming joints like hip joints. Although artificial joints produce a range of particle sizes, conforming joints such as hip replacements produce a number of smaller particles greater than the number of larger particles produced by nonconforming total knee replacements. 


\subsection{Reducing surface damage}

By minimizing the stresses associated with the types of damage that can occur or by increasing the strength of the polyethylene, the risk of surface damage can be decreased. We know that abrasive wear which occurs in hip joints can be minimized by a reduction in contact stress. Interestingly, both the range of the maximum principal stress and the maximum shear stress generally decrease when contact stress is decreased. Therefore, one can reduce the risk of abrasive wear and pitting and delamination by reducing the contact stress. The overall design goal is to choose the geometry of the articulating surfaces and material properties of the polyethylene that minimize contact stress.

\subsubsection{Minimizing contact stress}

Contact stresses in acetabular components are affected by changes in loading, conformity of the articulating surfaces, thickness of the polyethylene, and stiffness of the material. Contact stresses increase with increasing load. If the same prosthesis (same conformity, thickness, and material) is used in patients with different weights, the stresses will be higher in the heavier patients. The stresses are not directly proportional to the load. As the load between the contacting surfaces increases, the contact area also gets larger. The contact stresses, of course, are not uniform over the contact area. The metal component of a total joint replacement is a rigid indenter. The contact stress will be greatest where the surface displacement of the polyethylene is greatest. Consequently, the displacement of the polyethylene surface in a direction normal to the surface will be determined by the shapes of the two contacting surfaces. For example, if the indenter and the polyethylene are both spherical, as they are in an ideal total hip replacement, then the maximum displacement of the polyethylene will occur at the center of contact. Therefore, the maximum contact stress will occur at the center of the contact area, and the minimum contact stress (zero) will occur at the edge of contact. Furthermore, the shape of the contact area in this ideal case will be circular. If the surfaces are not spherical because of either design or manufacturing variations, then the maximum contact stress may not be at the center of the contact area. The acetabular surface has ripples in it. As a result, when the femoral head is pressed into the polyethylene, the largest displacement normal to the surface of the polyethylene component will be at the apexes of the ripples. Therefore, the greatest contact stresses will also occur at these points. The stress in the valleys in the contact area will be small, because the deformation (difference between the dashed and solid lines) will be small at these points. Surface waviness can be caused by normal variations in manufacturing processes. Changes in conformity, thickness, and material properties cause changes in the contact area. In general, changes that decrease the contact area will increase the stresses, because the same load must be distributed over a smaller region. The contact area decreases when the conformity between the articulating surfaces decreases, when the thickness of the material decreases, and when the stiffness of the material increases. The effects of changes in thickness and elastic modulus on contact stresses may be understood as follows. Conceptually, the polyethylene may be considered to be supporting the metal indenter by a collection of parallel rods that are aligned along the direction of loading. Each rod supports a portion, $\delta P$, of the total load $P$. The stiffness of a rod under axial load is given by

$$
\mathrm{K}_{\text {rod }}=\delta \mathrm{P} / \Delta=\mathrm{EA} / \mathrm{L}
$$


where $\Delta$ is the displacement of the rod, $\mathrm{E}$ is the elastic modulus of the rod material, $\mathrm{A}$ is the cross-sectional area of the rod, and L is the length of the rod, which corresponds to the thickness of the polyethylene component. It can be seen from this equation that the structural stiffness of the rod increases as the length of the rod decreases. In a similar way, the structural stiffness of polyethylene components increases with decreasing thickness. When the stiffness of the component increases, the indenter does not displace as much, the contact area decreases, and the contact stresses increase. The rod analogy further shows that structural stiffness also increases when the elastic modulus of the rod material increases. Similarly, the structural stiffness of a polyethylene component increases when the elastic modulus of the polyethylene increases, leading to a decrease in contact area and an increase in contact stress. The contact stresses for acetabular components are within the elastic limit of the polyethylene. For perfectly conforming spherical contact, it can be shown that the contact stresses are independent of cup thickness and modulus. This is consistent with observations of in vivo head penetration rates, which are constant with time after an initial wearing-in period. On the basis of contact stress alone, larger head sizes would be preferred. But it has been shown that wear at a point is proportional to pressure times sliding distance. Consequently, there is a trade-off between the relative sliding of the contacting surfaces and the contact stress, both of which are a function of diameter. The maximum shear stress in polyethylene acetabular components occurs very close to the articulating surface and this fact has not yet been directly linked to the wear seen in these components.(Teoh 2002)

\subsection{Particle debris}

The generation of particulate debris is a central focus of attention in the arthroplasty literature. The biologic response to wear debris is currently heralded as the single most important factor limiting the long-term durability of contemporary total hip and total knee replacement arthroplasty.

\subsubsection{Types of particles}

Current data suggest that tissues adjacent to a failed joint prosthesis contain billions of particles per gram of tissue. A wide variety of particle types have been retrieved from periprosthetic tissues at the time of autopsy or revision, as well as from joint simulators. In general, these particles types may be classified as metallic, polymeric, and ceramic. The majority of reports on periprosthetic metal debris pertain to $\mathrm{Co}-\mathrm{Cr}$ and titanium. Metallic particles are characteristically gray to black, and although they may appear weakly birefringent under polarized microscopy, the appearance of birefringence is an optical artifact because the particles are actually opaque. The metal particles are generally smaller than polymer particles but larger than ceramic particles.

Submicrometer metallic particles have been described as globular or irregularly shaped as well as elongated with sharp corners. Larger Co-Cr particles, in the 1- to 5- $\mu \mathrm{m}$ size range, have been described more often as needle, rod, or splinter shaped. Even larger Co-Cr particles, from $5 \mu \mathrm{m}$ to over $1 \mathrm{~mm}$, have been described as irregularly shaped or globular. These particles tend to be extracellular and may actually represent aggregates or clusters of smaller particles. The majority of titanium particles likewise range from under a micrometer to less than $5 \mu \mathrm{m}$ in size and have been described as blackish-gray material and fine powder. Occasional titanium particles from $5 \mu \mathrm{m}$ up to $1 \mathrm{~mm}$ in size have been reported. (Malviya 2010) 
The most common polymer particles encountered in association with joint prostheses are PMMA and UHMWPE particles. PMMA, unlike UHMWPE, is normally not birefringent. There are several potential sources of PMMA particles, including intraoperative debris, fatigue failure of cement and fretting of bone-cement and prosthesis-cement interfaces. In addition, unconsolidated or poorly mixed PMMA may release 25- to $35-\mu \mathrm{m}$ "prepolymerized spheres". Certain elements of tissue processing for light microscopy dissolve PMMA and therefore it may be represented by histologic voids, ranging from less than $1 \mu \mathrm{m}$ to greater than $1 \mathrm{~mm}$ in size. The particles or voids are irregularly shaped and have been described as multifaceted or resembling slivers of glass. Barium sulfate or zirconium oxide which have been added to most PMMA since the 1970s to permit visualization of the cement on radiographs has been reported in tissue voids left by PMMA. UHMWPE debris is translucent and strongly birefringent under polarized light microscopy. The particles from the femoral area of aseptically loose femoral components are predominantly spherical or globular in shape, ranging from 0.1 to $1 \mu \mathrm{m}$ with a mean of 0.5 $\mu \mathrm{m}$. Over $90 \%$ of these particles are less than $1 \mu \mathrm{m}$ in size. The spherical particles are also associated with fibrillar attachments either singly or forming aggregates of fine particles. The fibrils ranged from 0.3 to $1.0 \mu \mathrm{m}$ wide to 10 to $25 \mu \mathrm{m}$ in length. Debris over $100 \mu \mathrm{m}$ in length have been described as shredded fibers and flattened UHMWPE rolled in the articulation and resembling cigars. There has been great recent interest in the use of highly cross-linked UHMWPE in total joint arthroplasty as a means of decreasing the wear rates of the polyethylene bearing surfaces. It is known that the mechanical properties of UHMWPE are directly related to its molecular weight, crystalline ultrastructure, chemical structure and thermal history. The increased intramolecular cross-links of highly cross-linked UHMWPE are thought to better resist deformation and wear. The wear particles from highly crosslinked polyethylene are smaller (often in the submicrometer and nanometer range) than those from conventional UHMWPE. Controversy exists regarding the ability of these submicrometer particles to generate the inflammatory response that ultimately results in osteolysis. It is well known that particle morphology can strongly influence biological response. The elongated particles of UHMWPE were more biologically active than globular particles.(Gul 2003)

A resurgence of interest in ceramic articulating surfaces has been driven by various reports of their excellent wear characteristics. Still, ceramic wear debris is reported and particles are most commonly 1 to $5 \mu \mathrm{m}$ in size. In a study of tissue retrieved at the time of revision, Hatton et al. found a bimodal distribution of sizes of ceramic wear debris. Using low resolution electron microscope, ceramic particles between 0.05 and $3.2 \mu \mathrm{m}$ were found. Using high resolution electron microscope, particles between 5 and $90 \mathrm{~nm}$ were visualized. It was theorized that the larger particles were generated from microseparation and impaction of the ceramic surfaces and that the smaller particles were generated from normal articulation of the bearing surfaces.( Hatton 2002b) While often thought of as inert, ceramic wear debris may be able to generate a biologic response that leads to osteolysis. Many studies have shown that ceramic particles can incite inflammatory and cytotoxic effects. Hatton et al. demonstrated that tissues from around ceramic-ceramic hips had areas rich in macrophages, large amounts of neutrophils and lymphocytes, and areas with up to $60 \%$ necrosis. The control group, tissue from Charnley metal-on-poly hips, showed the presence of giant cells and a dense macrophage infiltrate, but there was less than $30 \%$ necrosis. There were significantly more neutrophils in the ceramic-ceramic tissues and significantly more macrophages and giant cells in the metal-on-poly group.(Hatton 2002b; Hannouche 2005) 
In certain clinical settings, relatively large numbers of particles of corrosion products have also been identified. Chromium-orthophosphate-hydrate-rich particles were noted at the modular prosthetic head-neck junction, in the UHMWPE liner, and in the pseudocapsule around the prosthesis. In fact, in certain cases, chromium orthophosphate was second only to UHMWPE in number of intracellular particles found in periprosthetic tissues. The vast majority of chromium orthophosphate particles are less than $5 \mu \mathrm{m}$, and they are described as noncrystalline, translucent, and colorless. In addition to particles arising from the prosthesis or cement, certain anomalous particles have also been described. Silica has been reported in interfacial membranes, most likely a remnant of the catalyst used in UHMWPE manufacturing or a remnant of the sandblasting of metal prosthetic components. These are likely sources of trace amounts of aluminum as well. Furthermore, iron- and nickelcontaining particles (stainless steel) were likely contaminants from surgical instruments. Calcium- and phosphorous-rich particles were noted and, judging from the ratio of their concentrations, were probably from bone mineral. Thus, bone particles are also present in the periprosthetic milieu.(Jacob 2006)

\subsection{Osteolysis}

Osteolysis is a significant cause of aseptic loosening and is the biggest cause of revision surgery. It is due to resorption of bone, which is seen more commonly around the acetabulum than the femur. There is a link between wear and corrosive debris formation and osteolysis and is seen more commonly in patients with high wear of their implants. It is often seen as lucency around the implants on radiographs taken years after the original procedure, but can be underestimated on plain films. The primary cellular mediator is the macrophage. In the presence of debris it produces a number of cytokines and inflammatory mediators (IL-1, IL-6, IL-10, TNF-a and prostaglandins). These initiate increased osteoclast activity and also increase osteoclastic differentiation, whilst the debris particles actually have a detrimental effect on osteoblastic bone formation. (Howcroft 2008) Within osteolytic areas other cell lineages may be found including fibroblasts and lymphocytes. Both of these contribute to the ongoing osteoclastic activity and periprosthetic bone resorption.(Archibeck 2000) It is now becoming evident that the size of the wear particles is of equal importance to their number. Nanometer particulate wear debris does not appear to affect the osteoblasts significantly, and coupled with a lower wear rate longer implant survival could be predicted. As the bearing surfaces clearly have an effect on implant survival, it is important to consider the different pairings and look at the relative merits of each. (Harris 2001)

\subsection{Clicking and squeaking}

With the aid of fluoroscopy, articular surfaces separation during the hip motion has been observed. During this hip separation there is a loss of contact area, leaving only edge contact. Although separation has been well documented, it has not been correlated to clinical complications nor has a more in-depth understanding of the cause and effect been developed. Glaser et al studied the correlation between hip separation and sound production in different bearing surfaces.(Glaser 2008) In their study, among all analyzed subjects, the patients with a metal-on-polyethylene articulating surface experienced the highest magnitude of separation. The ceramic-on-polyethylene group had, on average, relatively low separation values, and these subjects demonstrated much less jerky motion. The maximum separation for the metal-on-metal polyethylene sandwich subjects was 1.5 
$\mathrm{mm}$. Interestingly, in the metal-on-metal group with large femoral heads (52 $\mathrm{mm})$, all patients experienced very high separation values after heel-strike in the first $30 \%$ of stance phase, which decreased to a no-separation condition during midstance. In the metal-onmetal group with small-size femoral heads $(38 \mathrm{~mm})$, the trend was similar to the other bearings with small femoral heads.(Komistek 2002) For all patients, the sound signals were examined and compared with the kinematic findings. Interestingly, there was a distinct correlation of a high-frequency sound occurring at the time when the femoral head slid back into the acetabular component. As the femoral component impacted the acetabular cup, the sound sensor revealed a high-frequency sound, representing impact conditions. A thud-like "clicking" sound was detected for the subject having a metal-on-polyethylene bearing. Similar, but much more accentuated, was the sound recorded for the subjects with a ceramic-on-polyethylene prosthesis. Clear and rich "clicking," combined with some crepitus, was observed for the subjects having a metal-on-metal polyethylene sandwich total hip arthroplasty. The subjects with a metal-on-metal total hip prosthesis experienced a sound similar to a "rusty door hinge". Ceramic-on-ceramic total hip arthroplasty subjects experienced a "squeaking" sound of variable degree, which was present throughout the entire gait cycle. The ceramic-on-ceramic articulations were considered to be the noisiest.(Rodriguez 2008) In all patients with separation, a knocking sound was observed when the femoral head contacted the acetabulum.(Glaser 2008)

Mismatched ceramic couples, acetabular component malposition and impingement have been proposed as factors in the development of squeaking. However, not all mismatched and malpositioned components lead to squeaking. (Restrepo 2008) Additionally, squeaking has been observed in properly matched and positioned implants and when no evidence of neck-to-rim impingement is present. Audible squeaking of a hip replacement remains a still unexplained phenomenon. The sliding motion within the acetabular cup could lead to the induction of vibrational propagation across the interface of the femoral head and acetabular cup, possibly leading to audible interactions. It is also hypothesized that the "squeaking" sound, which mainly occurs in ceramic-on-ceramic total hip prostheses, may be due to separation of the femoral head. This movement of the femoral head from the acetabular component and the impact conditions generated from sliding back can be a source for the acoustic emission observed in total hip prostheses. (Jarrett 2009)

\section{Alternate bearing surfaces}

During total hip arthroplasty, both the femoral and acetabular bearing surfaces are surgically replaced with metallic, polymeric, and/or ceramic components.(Fig.6) Throughout the twentieth century, many different combinations of these materials have been explored as candidate bearing surfaces for total hip arthroplasty. Today the most widely accepted bearing couple (i.e., combination of bearing materials for the hip joint) consists of a femoral head fabricated from cobalt chromium molybdenum (CoCr) alloy articulating against a polymeric component fabricated from ultrahigh molecular weight polyethylene (UHMWPE). The use of the CoCr/UHMWPE bearing couple has provided consistent results in total hip arthroplasties around the world for the past four decades. In 1998, an estimated 1.4 million UHMWPE components were implanted worldwide, with approximately half of these bearings being implanted in the hip. At most 200,000 metal-onmetal or ceramic-on-ceramic components have been implanted in patients worldwide between 1988 and 2000, corresponding to less than 10\% of total hip replacements during the 
same time period. Despite the recognized success and worldwide acceptance of total hip arthroplasty, wear of the UHMWPE component is a major obstacle limiting the longevity of these reconstructions.

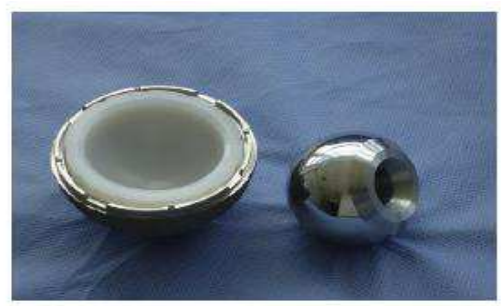

A

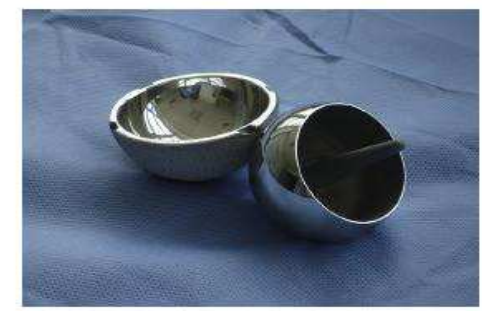

B

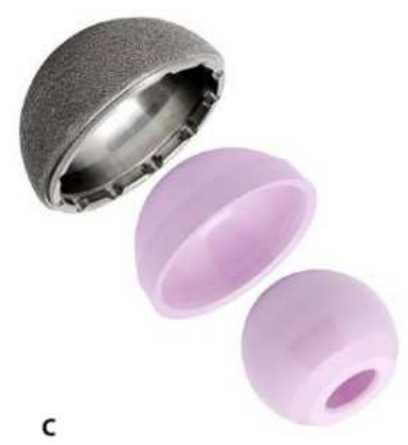

Fig. 6. Different current bearing surface options; A, metal-on-polyethylene, B, metal-onmetal, and C, ceramic-on-ceramic

\subsection{Materials bearing against polyethylene}

\subsubsection{Metal-on-polyethylene bearings}

The metals used in conjunction with polyethylene principally have included stainless steel, cobalt-chromium alloy (in the vast majority), and titanium alloy. In some cases, the metal components have been surface hardened; for example, by nitriding or ion-implanting. In general, the wear rate of polyethylene against stainless steel has been comparable to that against cobalt-chrome alloy in laboratory tests and in clinical use. In contrast, although the wear rate of polyethylene against titanium alloy under clean conditions appears to be comparable to that with the other metals, the greater vulnerability of titanium alloy to abrasion by entrapped third-body particles can cause severe, runaway wear. Hardening of the surface of the titanium alloy by techniques such as gas nitriding, solution nitriding, or ion implanting can markedly improve its resistance to abrasion by third-body particles, and good 10-year results have been reported for titanium nitride-hardened TiAlNb alloy balls used with polyethylene cups. Nevertheless, if a hardened surface eventually is penetrated, severe wear of the underlying alloy still can be triggered. Consequently, even hardened titanium alloys have seen limited clinical use as bearing surfaces. The vast majority of metalon-polyethylene bearings used in hip prostheses have involved cobalt-chrome alloy femoral balls, including cast or forged alloys, and the wear rate of this combination now forms the clinical baseline against which potentially improved bearing combinations are evaluated. As 
noted above, the average wear rate of the polyethylene against cobalt-chrome alloy is typically reported to be in the range of 0.1 to $0.2 \mathrm{~mm} / \mathrm{yr}$. However, it should be noted that this average includes those implants that have accelerated wear rates due to excessive thirdbody damage to the bearing surfaces, radiation-induced oxidative degradation of the polyethylene, or other causes. Thus, the inherent wear rate of a polyethylene cup with a cobalt-chrome alloy ball under clean conditions is probably somewhat below the clinical average wear rate, possibly as low as $0.05 \mathrm{~mm} / \mathrm{yr}$. Ion implanting and other surface hardening techniques also have been applied to cobalt-chromium alloy. Laboratory tests of hardened cobalt-chromium alloy have been reported to both markedly reduce wear and to increase wear of the opposing polyethylene, and clinical results are not yet sufficient to resolve this contradiction. Whereas it seems likely that surface hardening of cobalt-chrome may improve its resistance to moderate amounts of third-body abrasion, the uncertainty of the advantage in general has limited its clinical application.(Kim 2005)

\subsubsection{Ceramic-on-polyethylene bearings}

Alumina and zirconia femoral balls have been used widely as bearing surfaces against polyethylene cups, and most clinical studies have shown substantially lower polyethylene wear rates than with metal balls, with the wear ratios ranging from 0.75 to as low as 0.25 with alumina balls. A comparable advantage has been reported with zirconia against polyethylene.(Urban 2001) Unacceptably high rates of polyethylene wear, lysis, and loosening with an early type of zirconia ball were reported in a study. Similarly, although the majority of the laboratory tests have indicated lower wear of polyethylene with alumina or zirconia than with metal, in one hip simulator study slightly greater polyethylene wear was reported with alumina balls, but less with zirconia balls.(Yoshitomi 2009) The greater hardness of ceramic balls renders them more resistant than metal balls to scratching by entrapped abrasive contaminants that can, in turn, accelerate the wear of the opposing polyethylene cup (wear mode 3 ). The differences in the relative wear rates in the various clinical studies might reflect differences in the amount of third-body contamination, with those studies having relatively little such contamination showing comparable polyethylene wear rates for ceramic or metal balls. Nevertheless, contamination by metal particles may be detrimental even with a ceramic ball, because the particles can adhere to the surface of the ceramic, effectively roughening it and, thereby, increasing abrasion of the polyethylene. Metal also can be transferred to the ceramic by contact against metallic components or instruments during surgery.(Garvin 2009) Regardless of the bearing material used, care must be taken to minimize the formation of abrasive contaminants in vivo, for example, by avoiding those porous coatings that are prone to shed particles. (Clarke 2000)

\subsection{Polyethylenes}

\subsubsection{Chemical structure and molecular weight}

Polyethylene is a polymer of ethylene and consists of a carbon backbone chain with pendant hydrogen atoms. It is the simplest of polymer molecules chemically, but as the length of the polymer chain increases, so too does the complexity of the material. UHMWPE, used in orthopedic hip and knee applications since 1962, has a molecular weight ranging from 2 to 6 million daltons. By virtue of its molecular weight, UHMWPE has the desirable attributes of wear and impact resistance, together with ductility and toughness. These attributes make UHMWPE highly suitable as a bearing material. There has been some confusion in the 
historical arthroplasty literature, in which what is now considered UHMWPE was historically referred to as high-density polyethylene (HDPE) or polythene. Today, highdensity polyethylene refers to a material with a molecular weight of 100 to 250,000 daltons and is suitable for milk jugs, not artificial joints. In a hip simulator, HDPE has a wear rate that is four times higher than that of UHMWPE.

The ultra-high molecular weight polyethylenes (UHMWPEs) that were used for acetabular cups implanted during the past 30 years were fabricated from raw powder (also called resin). The powder is converted to solid form using one of three distinct methods. In the extrusion process, the polyethylene powder is driven by a ram through a heated nozzle, fusing the flakes into a continuous bar, typically several inches in diameter. The components are machined from the bar stock. In bulk compression molding, the powder is placed in a large mold and heated under pressure to fuse it into a block or sheet from which the final components are machined. In net-shape molding, the powder is placed in a metal mold having the desired shape of the implant and then fused under heat and pressure, such that little or no final machining is required. The final step in any fabrication process was sterilization.

\subsubsection{Crystallinity}

Crystallinity is an important attribute of all polyethylenes, including cross-linked polyethylene. The molecular chains in polyethylene have a natural tendency (driven by thermodynamics) to preferentially fold up against themselves whenever possible, hindered by the considerable crowding and thermal jostling presented by adjacent molecules. Regions of the polymer with folded chains are referred to as crystallites, whereas the regions with randomly oriented polymer chains are referred to as the amorphous regions. In polyethylene, the crystallites have a particular "lamella" shape. If we were to dissolve away the amorphous regions, the crystalline lamellae in polyethylene would look something like twisted, interconnected sheets. The molecular chains are oriented perpendicular to the plane of the lamella and may emerge to connect with adjacent lamellae.(Fig.7)

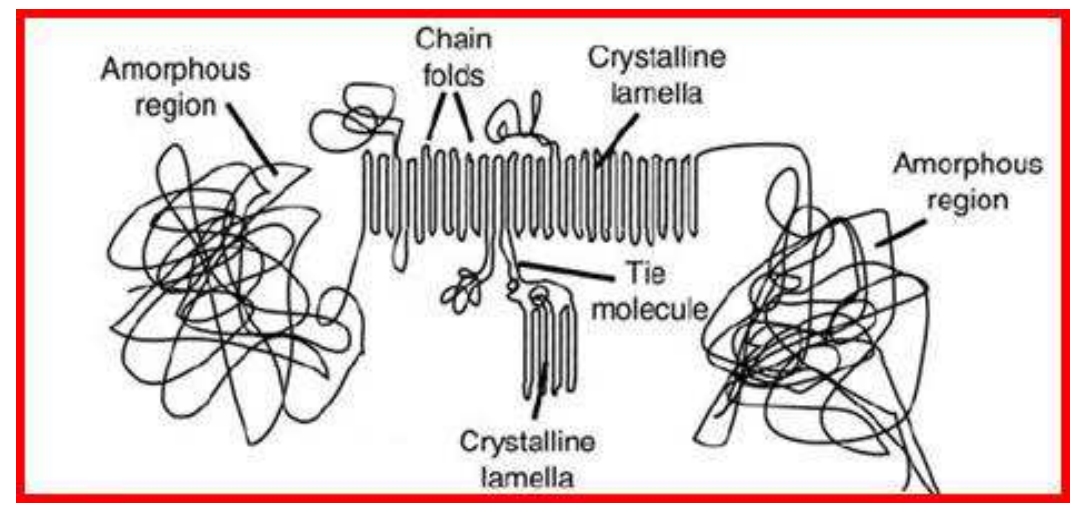

Fig. 7. Schematic crystalline structure of polyethylene

These connective polymer chains (not shown) are referred to as tie molecules. In particular, it is thought that tie molecules contribute greatly to the inherent wear resistance of polyethylene. The elastic modulus and yield stress of polyethylene will increase in direct 
relation to the number of crystals present. Many of the processing steps for clinical polyethylenes are tailored specifically to optimize the crystalline structure and thereby tune its material properties. Polyethylene typically has a crystalline content of about $50 \%$. The thermal processing alters the basic organization of molecular chains in polyethylene by modifying the size and shape of the crystals.

\subsubsection{Cross-linking}

Cross-linking is the foundation of all modern polyethylene total hip bearings. Cross-linking is defined as the joining of two independent polymer molecules by a chemical covalent bond. Only radiation cross-linking has been commercialized by orthopedic device manufacturers. The first step involves irradiation of the polyethylene molecule. Next, irradiation produces a hydrogen radical, leaving a so-called "free" radical on the polyethylene molecule. Actually, the radical on the polymer chain has extremely limited mobility and is hindered by the adjacent molecule. For cross-linking to occur, free radicals must be present on adjacent polyethylene molecules, and the molecules must be mobile. Free radical recombination takes place primarily in the amorphous phase of the polymer, where the molecules are in close enough proximity to allow the formation of the interchain carbon-carbon bond that constitutes the cross-link. When the adjacent radicals react, a covalent bond, or cross-link, is formed between the two polyethylene molecules. In the crystalline phase, because of the increased distance between the molecules, crosslinking is not favored. As a result, the free radicals generated in the crystalline regions are postulated not to take part in the crosslinking reactions and become trapped. These residual free radicals are the known precursors of oxidation-induced embrittlement secondary to gamma sterilization. (Muratoglu 2001)

The extent of cross-linking in polyethylene is proportional to the absorbed dose of radiation. Historically, polyethylene bearings were gamma sterilized at a dose of 25 to $40 \mathrm{kGy}$. This dose resulted in the formation of some cross-links. Saturation of cross-linking was achieved only at approximately $100 \mathrm{kGy}$ of absorbed dose. Today, cross-linked polyethylenes are processed with a total dose ranging from 50 to $105 \mathrm{kGy}$. In general, increasing the dose provides a proportional improvement in wear resistance, with diminished benefits observed above 100 kGy. (Geedink 2009)

\subsubsection{Thermal processing: Annealing and remelting}

In the production of a highly cross-linked UHMWPE, the material is subjected to a thermal treatment step to reduce the level of free radicals via further cross-linking reactions. At higher temperatures the polymer molecules have increased mobility, thereby increasing the probability of free radicals on adjacent chains to react and form cross-links.(Fig.8) For the thermal treatment to be effective at eliminating all free radicals, it must be conducted above the melting temperature of the material at $150^{\circ} \mathrm{C}$. Heating above the melting temperature destroys the crystalline regions of the material, thus making the free radicals that were in the crystals available for cross-linking. The disadvantage of melting is the reduction in polymer crystal size and in mechanical properties (e.g., material yield and ultimate strength) that ensues when the material returns to room temperature. A compromise solution is to heat the material to $130^{\circ} \mathrm{C}$ to $135^{\circ} \mathrm{C}$, just below the melting temperature. This solution preserves the original crystal structure, retains mechanical properties, and makes more free radicals available for cross-linking than would be available without thermal treatment. Some free 
radicals are retained in the crystal domains, but the number is substantially reduced by the elevated temperature. When thermal treatment is conducted below the melt transition of $135^{\circ} \mathrm{C}$, it is referred to as annealing, and above the melt transition, it is called remelting.

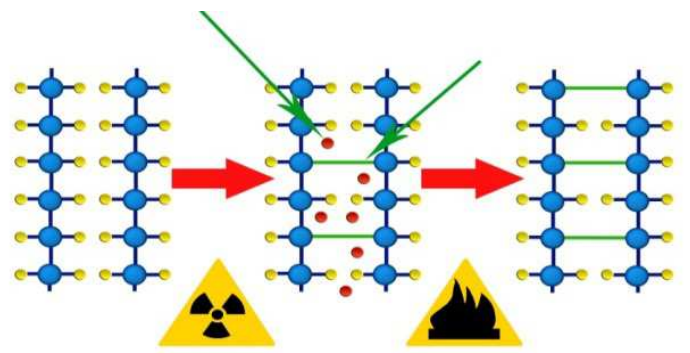

Fig. 8. Diagrammatic representation of the manufacturing process of highly cross-linked polyethylene; the effects of radiation and heat treatment

\subsubsection{The effect of crosslinking on mechanical properties of polyethylene}

The changes in the mechanical properties of the radiation- and heat-treated polyethylene are primarily dominated by changes in the crosslink density and crystallinity. Under multiaxial loading conditions, it is more difficult to separate effects of crystallinity and cross-linking, since both appear to influence the large-strain mechanical behavior in a more complex, synergistic manner. The crystallinity of polyethylene is a function of the radiation dose level and thermal treatment history. Irradiation generates smaller chains with increased mobility, leading to recrystallization and a slight increase in the crystallinity of the polymer. The changes in the crystallinity during the postirradiation thermal treatment depend on temperature. If the thermal treatment is carried out below the melting transition $\left(<135^{\circ} \mathrm{C}\right)$, the chain mobility increases, which, in turn, increases the crystallinity of the polymer. When the thermal treatment is performed at temperatures above the melting transition $\left(>135^{\circ} \mathrm{C}\right)$, during cool-down to room temperature, the crystallization of the polymer takes place in the presence of the crosslinks. This leads to a decrease in the crystallinity of the polymer. The radiation dose level used in the irradiation step determines the crosslink density. The crosslink density of the polyethylene limits the ultimate elongation that can be achieved during plastic deformation prior to failure. Therefore, at higher radiation dose levels, the cross-linked polymer exhibits reduced ultimate tensile strength and elongation at break under uniaxial tension. As a result, the work to failure also decreases. As the crosslinking reduces the chain mobility, it also inhibits the active energy-absorbing mechanisms. Therefore, at high uniaxial deformation rates, such as impact loading, the energy absorption before failure decreases, leading to a decrease in the toughness.(Baker 2003) Another important variable that affects the mechanical properties of the radiation- and heat-treated polyethylene is the irradiation temperature. When the polymer is irradiated at an elevated temperature $\left(90^{\circ} \mathrm{C}<\mathrm{T}<135^{\circ} \mathrm{C}\right)$ the effect of the crosslink density on the large strain mechanical properties decreases significantly. This may be explained by a nonstatistical distribution of the crosslinks resulting at increased irradiation temperatures. As a result, the low crosslink-density matrix controls the large-strain mechanical properties.(Dumbleton 2006) The polyethylenes irradiated at increased temperatures have been reported to exhibit 
better large-strain mechanical properties than those irradiated at room temperature with identical radiation dose levels.(Affatato 2005)

\subsubsection{Conventional polyethylene surfaces}

Although ultra-high-molecular-weight polyethylene (UHMWPE) has low friction and dampening properties, it has one major disadvantage, which is adhesive and abrasive wear. The UHMWPE particles produced from cyclical loading are thought to play a major role in particle-induced osteolysis and secondary implant failure and loosening. Osteolysis results from the intrusion of polyethylene debris between the implant surface and bone, inducing a macrophage response. Polyethylene also can fail as a result of third-body wear secondary to polyethylene degradation. A polyethylene wear rate of $0.10 \mathrm{~mm}$ per year is the threshold for the development of osteolysis. In addition, conventional polyethylene thickness should not decrease below 6 to $8 \mathrm{~mm}$, or accelerated wear polyethylene failure and osteolysis will develop.(Lundberga 2006)

\subsubsection{Highly cross-linked polyethylene surfaces}

Highly cross-linked polyethylene was introduced to reduce the polyethylene wear rate in THA.(McKellop 1999) It is well documented that young age, male gender, and high activity level increase the risk of wear, osteolysis, and mechanical failure. However, these different factors that influence wear rate in conventional polyethylene have no significant influence when using highly cross-linked polyethylene.(Rohrl 2007) In vitro studies on highly crosslinked polyethylene have shown that wear can be reduced by $42 \%$ to $100 \%$ compared with that of conventional polyethylene, and, multiple in vivo studies support many of these in vitro results. The annual linear wear for highly cross-linked polyethylene has been reported to be $45 \%$ that of the conventional liner at 5 years after implantation. This wear rate is well below the threshold for lysis. Long-term results (10 to 20 years) of highly cross-linked polyethylene support that the low wear rates reported approach that of MOM and ceramicon-ceramic bearings.(Digas 2007; Atienza 2008)

\subsubsection{Sterilization techniques}

Today, polyethylenes are sterilized by different techniques with regard to the fact that sterilization process can affect the physical properties of polyethylene. Some brands of the polyethylene components are now sterilized without irradiation, using either ethylene oxide or gas plasma, in order to minimize the oxidative degradation. Because these methods do not generate free radicals in the polyethylene, they completely avoid the potential for immediate and long-term oxidative degradation of the mechanical properties and wear resistance. However, because ethylene oxide or gas-plasma do not induce cross-linking, these polyethylenes do not take advantage of improving the wear resistance following crosslinking.(Digas 2003) Other brands of polyethylenes are still sterilized with gamma radiation, but for doing so, the polyethylene components are sealed in some type of low-oxygen atmosphere, including vacuum, inert gas, or with an oxygen scavenger. In addition, one manufacturer anneals the polyethylene acetabular cups after sterilization by heating them in the nitrogen packaging at $37^{\circ}$ to $50^{\circ} \mathrm{C}$ (well below the melt temperature of $135^{\circ} \mathrm{C}$ to avoid distorting the components) for about 6 days to reduce the level of residual free radicals that were induced by the gamma radiation. These modifications in sterilization techniques can markedly reduce but not necessarily eliminate the oxidation that would otherwise occur 
during gamma sterilization and subsequent shelf-storage, and the remaining free radicals may induce some oxidation during long-term use in vivo. The moderate level of crosslinking that was present in the vast majority of UHMWPE components implanted over the past three decades was an unintentional byproduct of the 2.5 to 4 Mrads of gamma radiation used to sterilize the components. The improvement in wear resistance in these polyethylenes relative to non-cross-linked polyethylene ranges from about $30 \%$ to $50 \%$, compared to the $85 \%$ or more reduction achieved with intentionally elevated cross-linking doses. A number of laboratory wear simulations have demonstrated that the wear rate of UHMWPE cups decreases markedly with an increasing level of radiation-induced crosslinking. The greatest reduction per Mrad occurs as the dose increased from zero to about 8 Mrads, with progressively less improvement at higher doses and no additional benefit after 15 to 20 Mrads. While this dose-wear relationship was the basis for the recent development of a variety of intentionally-cross-linked polyethylenes, the developers have arrived at very different opinions regarding the appropriate dose and other processing parameters for optimizing the clinical performance of a polyethylene implant.

\subsubsection{Optimum method for cross-linking}

In addition to the optimum method of cross-linking, the optimum amount of cross-linking to use is also a subject of current debate. Because increasing the level of crosslinking causes a progressively greater reduction in some mechanical properties, such as ultimate strength, ductility, fracture toughness, and fatigue strength, one extreme is to avoid cross-linking altogether (for example, by simply sterilizing with ethylene oxide or gas plasma). This avoidance is to retain the maximum values of strength, elongation, and fracture toughness, despite the fact that it results in substantially higher wear of the polyethylene. Among those who advocate crosslinking, the particular dose used represents that manufacturer's approach to balancing reduced wear against the need to maintain other mechanical properties well above that needed for acceptable clinical performance. Those using the high levels of cross-linking (9.5 to 11 Mrads), about 3 to 4 times the typical dose used historically to sterilize polyethylene components, maintain that these high levels are justified to obtain the additional $5 \%$ to $10 \%$ improvement in wear over that provided by a moderate dose, despite the corresponding reduction in other physical properties. In contrast, advocates of a moderate cross-linking dose, such as 5 Mrads, maintain that the corresponding reduction in wear to $85 \%$ below that of a noncross-linked polyethylene will be sufficient to avoid an osteolytic reaction in even the most active patients, without unnecessarily reducing other physical properties. Clearly, it is not desirable to use a dose that will result in mechanical failure of the polyethylene components.(Moore 2008; Bradford 2004)

\subsection{Metal-on-metal bearings}

The first widely used total hip replacements featured cobalt-chromium alloy bearing against itself, primarily the McKee-Farrar (Howmedica, Limerick, Ireland) design, along with the Mueller (Sulzer AG, Winterthur, Switzerland), Ring (Downs, Ltd, Mitchham, England) and others. Because of a relatively high rate of early failure, the first-generation metal-on-metal hips were largely supplanted by the Charnley prosthesis, which featured a stainless steel ball and a polyethylene socket. Disregarding the early failures, the long-term survivorship of the early metal-on-metal designs has been comparable to that of the metal-onpolyethylene Charnley. In particular, the steady-state wear rates have been on the order of a 
few micrometers per year compared with the average of 100 to $200 / \mu \mathrm{m}$ of polyethylene wear per year typically reported for metal-on-polyethylene hips. In view of the growing awareness of the problem of extensive osteolysis caused by polyethylene wear debris, a number of second-generation metal-on-metal implants have been developed, including conventional total hips and surface replacements. The first to be widely used clinically was the Metasul (Sulzer AG, Winterthur, Switzerland) hip. Hip simulator studies and clinical retrievals of modern metal-on-metal bearings also typically have shown steady-state wear rates on the order of a few micrometers per million cycles (with one million cycles being the equivalent of about 1 year's use in a patient of average activity). It is also apparent that metal-on-metal implants have the ability to self-heal, that is, to polish-out isolated surface scratches caused by third-body particles or subluxation damage. The overall clinical performance of second-generation metal-on-metal hips to date has been comparable to that of conventional metal-on-polyethylene hips. Clinical and laboratory wear studies have indicated that metal-on-metal implants often exhibit 10 to 20 times greater wear-in during the initial 1 to 2 years of clinical use, or one to two million cycles in a hip simulator. In addition, some first-generation and second-generation metal-on-metal hips have exhibited extensive surface micropitting, possibly due to a fatigue-corrosion mechanism associated with the smaller carbides.

\subsubsection{Tribology}

The principle constituents of alloys for MOM bearings are cobalt, chromium, and molybdenum, but they can be differentiated as being either high carbon $(0.20 \%$ to $0.25 \%)$ or low carbon $(0.05 \%$ to $0.08 \%)$, with the former usually demonstrating lower in vitro wear rates. MOM hip bearings produce wear particles by some combination of the four classic mechanisms of adhesion, abrasion, corrosion, and surface fatigue, with an emphasis on abrasion and surface fatigue.(Wimmer 2003) Wear in metal-on-metal total hip arthroplasty implants is known to consist of two distinct phases. A relatively high-wear running-in phase, which lasts for between 0.5 and $2 \times 10^{6}$ cycles, is followed by a steady-state phase, during which the wear rate is constant and much lower. This decrease in wear has been demonstrated to be secondary to the so-called self-polishing effect of metal-on-metal bearing surfaces. Opposing metallic bearing surfaces are thought to run-in in the contact zone as surface asperities are polished or removed, improving the initial surface finish, with a commensurate reduction in the wear rate as the lubricating fluid film becomes more coherent. Metal-on-metal total hip arthroplasty implants can operate in the mild mixedlubrication regime in which much of the applied load is supported by elastohydrodynamic films. Correct tribological design leads to remarkably low steady-state wear rates. Promotion of the most effective elastohydrodynamic films calls for the largest possible head diameters and the smallest clearances that can be reasonably adopted consistent with fine surface finishes, good sphericity, polar contact, and minimal structural elastic deformation of the cup on its foundations.(Udofia 2003) With current manufacturing techniques, a surface roughness of 5 to $15 \mathrm{~nm}$ is typically achieved. This results in improved sphericity and surface finish. The presence of depressions or holes (rather than scratches) with smooth (rather than sharp) edges seems to improve the lubrication and wettability properties. The wear of metal-on-metal bearings is affected by various design parameters, such as the clearance or surface roughness. The predictive role of the $\lambda$ ratio in the evaluation of wear behavior of metal-on-metal total hip replacements has also been well documented. In an in 
vitro study, Affatato et al. demonstrated that femoral heads of $36 \mathrm{~mm}$ in diameter work in the mixed-lubrication regime $(\lambda>1)$ and 28 -mm diameter heads were associated with a $\lambda$ ratio of $<1$, thus showing their aptitude to work in the boundary-lubrication regime, with substantially higher volume depletion due to wear. With the 32-mm heads, the $\lambda$ ratio varied in a range around 1, suggesting the possibility of their operating in the mixedlubrication regime.(Affatato 2008) Hip-joint-simulator tests on metal-on-metal total hip replacements have shown a transition from boundary to mixed lubrication as the diameter of the femoral head increased. An increase in diameter up to $54 \mathrm{~mm}$ resulted in a marked reduction in wear rates, which was attributable to growing support from the fluid-film action in a mixed-lubrication regime. Reducing the clearance between the femoral and acetabular components also reduces the amount of wear debris. Significant reduction in the wear debris associated with large-diameter $(54-\mathrm{mm})$ heads has been noted when clearances were reduced. When clearances are designed, cup flexibility needs to be taken into account so that the minimum clearance is always more than the maximum flexibility of the cup.

Thick ( 8 to $12-\mathrm{mm})$ chromium nitride $(\mathrm{CrN})$ and chromium carbon nitride $(\mathrm{CrCN})$ coating leads to twenty-two-fold lower wear rates as compared with those associated with standard metal-on-metal couples. Also, $\mathrm{CrN}$ wear particles were found to be less cytotoxic than standard metal-on-metal bearings when co-cultured with macrophage and fibroblast cells. The substantially lower ion concentration released by these surface-engineered components along with the reduced wear volumes and the reduced cytotoxicity per unit volume of wear points to the potential for clinical application of this technology. However, there is concern about the durability of this coating.(Williams 2004)

\subsubsection{Materials and design considerations}

The interplay of material(s), macrogeometry (diameter and clearance), microgeometry (surface topography), and lubrication influences the wear of MOM-bearing THRs to a far greater degree than MOP-bearing ones. Alloys of cobalt $(\mathrm{Co})$ and chromium $(\mathrm{Cr})$ have been preferred for MOM bearings in THR because of their hardness. High chromium content provides good corrosion resistance. Cast Co-Cr-Mo alloy, which was used to manufacture the majority of first-generation MOM hips, has a relatively high carbon content of $0.2 \%$ to $0.3 \%$ and contains primarily $\mathrm{Cr}$ and Mo carbides, which result in asperities on the polished surface. The macrogeometry of a MOM bearing can be described in terms of the diameter(s) of the ball and the socket and the clearance of the resultant bearing couple. Clearance is the size of the gap between the surfaces at the equator of the bearing. For hemispherical bearings, clearance is a function of the difference in the diameters of the surfaces of the ball and socket. A ball and socket of exactly equal diameter mated together would have zero clearance and a maximal contact area for that size bearing. Contact area can be increased by increasing the size (diameter) of the bearing surfaces and/or by decreasing the clearance. Conversely, for a given diameter, increasing clearance decreases contact area. Contact stresses are a function of material properties and are inversely proportional to contact area. Clearance also influences lubrication, as the size of the gap has implications for the amount and type of lubrication. Smaller clearances encourage fluid film lubrication. Large clearances lead to a reduced contact area, loss of effective lubrication, and more rapid wear.(Reiker 2005) However, too little clearance may lead to equatorial contact, very high frictional forces, high torque, and loosening of the implant.(Chan 1996) 
The limitations of current mass production manufacturing set the lower limit of clearance at about $20 \mu \mathrm{m}$. Wear rate, particularly during the initial run-in, increases rapidly with clearances above about $150 \mu \mathrm{m}$. In addition to the contact area, another important variable is where the contact occurs. Given bearings of equivalent diameters, equatorial contact is associated with higher frictional torques than the same contact area in a more polar location. Equatorial bearing may have been a factor associated with failure of some early MOM THRs, and this is supported by retrieval studies. Consequently, relatively polar contact is preferred.(Dowson 2004; Isaac 2006) (Fig.9)
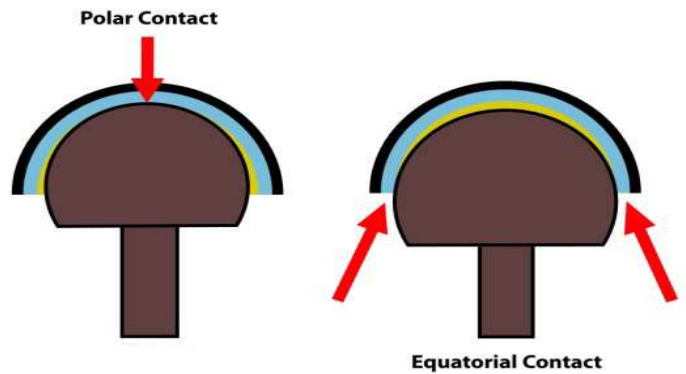

Fig. 9. Too much reduction in the "clearance" can result in conversion a polar contact to an equatorial contact resulting in the articulation seizing

\subsubsection{Lubrication}

The lubricating fluid and conditions are important variables which influence friction and wear. The synovial fluid of normal, osteoarthritic, and rheumatoid joints has been characterized to some degree. The fluid is thixotropic; viscosity is a function of the shear strain rate, or velocity of motion, for practical purposes. Less is known about the fluid formed around total joint arthroplasties, but the composition and rheology are likely variable. A favorable film thickness to surface roughness ratio ( $\lambda$ ratio) is desirable in order to maintain low friction between the articulating surfaces. Mixed-film lubrication appears to be the operative mechanism in most MOM hip joints. For a given load and surface velocity, fluid film thickness is dependent on the properties of the fluid but can be influenced by the properties of the bearing materials, the macrogeometry of the bearing (which is a function of diameter and clearance), and the surface microtopography (surface finish). (Affatato 2008) Within the apparent contact area, the surfaces make actual contact only at the tips of asperities, and a lubricant film can influence wear significantly. As wear proceeds, the contact area at the asperity tips increases, and such "running-in" can produce a more favorable microgeometry for lubricant films to separate the surfaces and reduce wear. Fluidfilm lubrication is encouraged by making the femoral head as large as practically possible and the clearance as small as practically possible. For MOM bearings, in distinction from PE bearings, larger diameters can actually produce lower wear rates for similar manufacturing parameters.(Smith 2001a; Chan 1999)

\subsubsection{Composition and microstructure of $\mathrm{CoCr}$ alloys}

$\mathrm{CoCr}$ alloys consist of a primary cobalt alloy matrix phase and a secondary metal carbide phase. In the cobalt-based matrix, chromium enhances the mechanical properties of the alloy 
and promotes the formation of a passive oxide layer, whereas molybdenum is included in the alloy for corrosion resistance. The size and distribution of the carbide phase contributes to the hardness and mechanical behavior of the alloy. The properties of $\mathrm{CoCr}$ alloys depend upon their processing history-whether fabricated by investment casting or by a thermomechanical forming process. During an investment casting process, a wax replica of the prosthesis is first fabricated and coated with a silicate slurry. The replica and slurry are raised above the melting point of the wax $\left(100-150^{\circ} \mathrm{C}\right)$ and burned away in a furnace, leaving only the slurry mold with an interior cavity having the dimensions of the replica. The cavity is then filled with molten alloy and, after cooling, the mold is broken to remove the cast implant. Mechanical forming, on the other hand, can be accomplished by a wide range of thermomechanical manufacturing methods, including rolling, forging, swaging, and drawing, all of which lead to a wrought $\mathrm{CoCr}$ alloy. Both cast and wrought CoCr alloys are subjected to annealing heat treatments for optimization of microstructure and mechanical properties. Historically, the first-generation metal-on-metal components were fabricated by casting(Hernandez-Rodriguez 2005), whereas contemporary secondgeneration metal-on-metal implants are manufactured from wrought $\mathrm{CoCr}$ alloys. (Barnes 2008)

Wrought $\mathrm{CoCr}$ alloys have significantly enhanced hardness, yield strength, and ultimate properties as compared with cast alloys, resulting from their more uniform carbide microstructure. All CoCr alloys used in orthopaedics contain trace constituents of carbon $(0.35 \%)$, nitrogen $(\max 0.25 \%)$, nickel $(\max 1 \%)$, iron $(\max 0.75 \%)$, silicon $(\max 1.0 \%)$, and manganese $(\max 1.0 \%)$. Cast $\mathrm{CoCr}$ alloys may also contain tungsten $(\max 0.20 \%)$, phosphorus $(\max 0.020 \%)$, sulfur $(\max 0.010 \%)$, aluminum $(\max 0.30 \%)$, and boron $(\max$ $0.01 \%$ ). Of these secondary constituents, the effect of carbon content on $\mathrm{CoCr}$ alloys has been examined most closely by researchers in the orthopaedic community because of the link between carbon content, carbide formation, and properties of the bearing surface. $\mathrm{CoCr}$ alloys may be further classified as either low-carbon $(<0.05 \%)$ or high-carbon $(>0.20 \%)$. The majority of contemporary metal-on-metal bearings are fabricated from a high-carbon $\mathrm{CoCr}$ alloy. In contemporary hip simulator studies, high- and low-carbon $\mathrm{CoCr}$ alloys have exhibited comparable wear mechanisms and wear rates. (Isaac 2006)

\subsubsection{Metal ions}

Ion levels have been measured in whole blood, serum, and erythrocytes. Metal ions from hip implant materials include the following: titanium; vanadium; chromium; cobalt; and molybdenum. In reports of metal ion concentrations in urine, for maximal accuracy and to minimize the effect of variations in the level of hydration that may occur throughout the day, a 24-hour urine collection should be performed. Healthy controls have been demonstrated to have a mean serum cobalt level of $0.24 \mathrm{mg} / \mathrm{L}$ (median, $0.23 \mathrm{mg} / \mathrm{L}$; range, 0.08 to $0.50 \mathrm{mg} / \mathrm{L}$ ) and a mean serum chromium level of $0.28 \mathrm{mg} / \mathrm{L}$ (median, $0.29 \mathrm{mg} / \mathrm{L}$; range, 0.06 to $0.93 \mathrm{mg} / \mathrm{L}$ ). Currently there are two methods for measuring trace metals: inductively coupled plasma mass spectroscopy (ICP-MS) and graphite furnace atomic absorption spectrophotometry (GFAAS), with the former method being favored because of higher sensitivity to detect low levels of metal ions, allowing multielement determinations.(Engh 2009) Numerous studies have reported an increase in the levels of metal ions in serum, urine, and blood of patients with MOM implants. However, serum/plasma levels of cobalt and chromium ions appeared to be higher than those in red blood cells, suggesting that serum may reflect better the true ion levels when using blood to 
assess systemic ion levels.(Daniel 2007; Antoniou 2008) In a study looking specifically at the serum cobalt concentrations in patients during the first 5 years after an MOM THA, Brodner and colleagues showed a moderate concentration of $1 \mu \mathrm{g} / \mathrm{L}$ at 1 year and 0.7 $\mu \mathrm{g} / \mathrm{L}$ at 5 years. They did not find any significant difference in the levels from 3 to 12 months and the subsequent measurements and, therefore, concluded that the serum cobalt concentrations did not reflect the higher run-in wear of MM implants.(Brodner 2003) In modern MM surface arthroplasty, Skipor and colleagues reported elevations in serum chromium that were 22-fold, 23-fold, and 21-fold higher at 3, 6, and 12 months postoperatively, respectively, than preoperatively. The corresponding serum cobalt levels were eightfold, sevenfold, and sixfold higher than preoperative levels, and the authors reported that the values seen with the current generation of surface arthroplasties were in the same range as those observed in association with conventional MOM THA.(Skipor 2002) Comparison of serum cobalt and chromium ion levels in patients with a MOM compared with a MOPE bearing and a MOM compared with a ceramic-ceramic bearing showed that there were always higher ion levels in the patients with the MOM bearings. In a study analyzing the whole blood cobalt and chromium ion levels in 68 patients, Hart and colleagues reported a decrease in cytotoxic CD8 ${ }^{+}$T-lymphocytes, which are involved in the defense against intracellular pathogens and cancerous cells.(Hart 2009) Of interest, Back and colleagues noted a significant decrease in the cobalt levels at 2 years compared with the levels at 6 months, as well as a decrease in the chromium levels at 2 years compared with the levels at 9 months. When looking at the effects of the size of the femoral head and the orientation of the acetabular component on the overall concentrations of metal ions in the whole blood of patients with MOM resurfacings, Langton et al. reported higher ion concentrations in patients with smaller components and when the inclination angle was greater than 45 degrees and the anteversion angle exceeded 20 degrees.(Langton 2008)

Wear particles from MOM bearings are nanometers in linear dimension, which is substantially smaller than PE wear particles. Light microscopic analysis of tissue obtained from around MOM joints showed particles with variable and irregular shape. The size of metal particles reported by scanning electron microscopy studies ranges from 0.1 to $5 \mu \mathrm{m}$. The studies have suggested that large metallic particles observed with light microscopy were agglomerates of the smaller particles. Transmission electron microscopy has demonstrated wear particles from Co-Cr-Mo bearings to be round to oval in shape with irregular boundaries. Most of the particles are smaller than $50 \mathrm{~nm}$ (range $6 \mathrm{~nm}$ to $1 \mu \mathrm{m}$ ). Additional analysis of these retrieved wear particles indicates that the particles have several different elemental compositions. There are Co-Cr-Mo particles, but there is an even greater number of chromium oxide particles. It has been hypothesized that the Co-Cr-Mo particles are produced by the wear of the carbides on the bearing surfaces and the prosthesis matrix, and that the chromium oxide particles come from the passivation layer on the implant surface and possibly from oxidized chromium carbides. There is little known about the rates of metallic particle production in vivo, lymphatic transport of metallic particles from the joint, and systemic dissemination. Utilizing information on volumetric wear rate and average particle size, it has been estimated that $6.7 \times 10^{12}$ to $2.5 \times 10^{14}$ metal particles are produced per year, which is 13 to 500 times the number of PE particles produced per year by a typical MOP joint. The aggregate surface area of these metal wear particles is substantial. (Malviya 2010) 
It is important to recognize that in modern THR prostheses there may be several sources of metal particle and ion generation.(Savarino 2006) Systemic dissemination of soluble and particulate corrosion products from modular junctions has been described, including the presence of metallic particles in the lymph nodes, liver, and spleen. In modular total hips with a MOP bearing, with no or mild corrosion at the modular head and neck junction, serum and urine levels of $\mathrm{Co}$ averaged $0.94 \mathrm{ppb}$ (range <0.54 to 1.65) and $0.92 \mathrm{ppb}$ (range $<0.3$ to 1.14), respectively, and urine $\mathrm{Cr}$ levels averaged $1.0 \mathrm{ppb}$ (range 0.54 to 1.92). With moderate or severe corrosion at the modular head and neck junction, the serum and urine levels of $\mathrm{Co}$ averaged $1.06 \mathrm{ppb}$ (range 0.8 to 1.4 ) and $0.87 \mathrm{ppb}$ (range $<0.3$ to 1.3), respectively, and the average urine Cr levels were $1.59 \mathrm{ppb}$ (range 0.6 to 3.0).(Jacobs 1998) The levels of metal ions in serum and urine are further elevated in patients with MOM bearings. It appears that the ion levels are higher in the short term and decrease over time. This is consistent with a conditioning phase or running-in of the bearing. The ion levels in subjects with Co-Cr alloy MOM THRs that had been in situ for an average of 24 years have been reported. The average serum Co level was $0.9 \mathrm{ppb}$ (range $<0.3$ to 2.0). The average serum $\mathrm{Cr}$ level was $1.28 \mathrm{ppb}$ (range 0.21 to 2.56), and the average urine $\mathrm{Cr}$ level was $1.22 \mathrm{ppb}$ (range 0.26 to 2.59).(Bitsch 2007) The longer-term serum and urine ion levels produced by MOM bearings are not much higher than those produced by the modular junctions of femoral components. Unfortunately, the toxicological importance of these trace metal elevations has not been established, and available data do not answer questions regarding the risks of ion hypersensitivity, toxicity, and carcinogenesis. Since wear of a MOM bearing cannot generally be measured on a radiograph, serum and urine metal ion concentrations may be useful indicators of patient activity and the tribological performance of these bearings.(MacDonald 2004) The distribution and histological effects of wear particles from a McKee-Farrar THR with an in vivo use of nearly 30 years has been described. Clinically, the implant was functioning well. Serum and urine levels of chromium and serum levels of cobalt, obtained 25 years after implantation and while the patient was healthy and active, were found to be $1.02 \mathrm{ppb}, 0.51 \mathrm{ppb}$, and $0.66 \mathrm{ppb}$, respectively. Sections from multiple samples of the lymph nodes, spleen, liver, and kidney were examined with light microscopy. This tissue analysis did not reveal any evidence of end-organ damage or accumulation of metal particles. It appears that a patient with normal renal function is capable of clearing cobalt and chromium ions from his or her system. Potential additional sources of ions include the acetabular morse taper connection.(Brodner 2003)

\subsubsection{Biological response}

Although the periprosthetic tissue reactions surrounding MOM THA demonstrate some histological characteristics similar to those typically seen around MOPE THAs, they have been reported to be less intense with fewer histiocytes present in the tissues. It is still uncertain whether this difference can be attributed to variations in particle concentration, size, shape, or composition. The wear particles produced by MOM implants in vitro and in vivo are in the nanometer size range, mostly round to oval but with the presence of some needle-shaped particles as well, mainly depending on the number of cycles (for in vitro particles) and the implantation time (for in vivo particles). In addition, most of the particles contain Chromium (Cr) and Oxygen (O) but no Cobalt (Co) and are therefore most likely chromium oxides. Therefore, in addition to focusing on CoCrMo particles, attention to the effects of chromium oxide particles should be addressed in future in vitro and in vivo biologic studies. Finally, because of their nanometer size, those particles have a high surface 
area and are subject to corrosion, releasing metal ions into the surrounding areas, and therefore the biologic response to metal ions also need to be considered.

The large aggregate surface area of metal wear particles may have both local and systemic effects. Surface area has been identified as a variable affecting the macrophage response to panicles. However, the local tissue reaction around MOM prosthesis, indicated by the number of histiocytes, is about one grade lower than that around MOP prostheses. A number of hypotheses have been proposed to explain this discrepancy. Since metal particles are considerably smaller than PE particles, histiocytes are able to store a larger number of metal particles, and therefore, the total number of histiocytes required to store the metal particles is lower. Very small particles may enter macrophages by pinocytosis instead of phagocytosis, which may alter the cellular response to the particles. It is also recognized that Co-Cr particles have greater potential for cytotoxicity than PE particles, and the cell may be incapable of the same inflammatory response. There may be a difference in the relative proportion of metal wear particles that are retained locally versus systemically distributed compared to PE wear particles. Dissolution of metal particles results in elevation of the cobalt and chromium ion concentrations in erythrocytes, serum, and urine. The metal particles have shown a dose-response effect. Low to moderate concentrations of metal particles stimulate the release of cytokines, such as interleukin-1 (IL-1), interleukin-6 (IL-6), tumor necrosis factor- $\alpha$, and prostaglandin E1, that can lead to periprosthetic osteolysis and aseptic loosening. At higher concentrations, however, $\mathrm{Co}-\mathrm{Cr}$ particles have been found to be cytotoxic, altering the phagocytic activity of macrophages and leading to cell death.(Kwon 2009; Huber 2010)

Although the volume of reactive periprosthetic inflammatory tissue associated with MOM bearings is less than with MOP, osteolysis can occur in hips with MOM bearings. The incidence of osteolysis associated with MOM bearings has not been well established but appears to be comparatively low.(Bharma 2006)

\subsubsection{Inflammatory versus immunological responses}

Because metal ions remain a major cause for concern in the widespread clinical use of MOM implants, in vitro studies have also been conducted on the effects of these metal ions. $\mathrm{Co}^{2+}$ and $\mathrm{Cr}^{3+}$ have been demonstrated to induce tumor necrosis factor (TNF)- $\alpha$ secretion. Petit and colleagues examined the effect of $\mathrm{Co}^{2+}$ and $\mathrm{Cr}^{3+}$ ions on protein oxidation in human U937 macrophages and showed that both $\mathrm{Co}^{2+}$ and $\mathrm{Cr}^{3+}$ ions induced a time- and dosedependent protein oxidation, reaching 6.5 and 2.9 times the control levels after 72 hours, respectively.(Bagchi 2002) A histological study of tissues surrounding MOPE and MOM THAs by Campbell and colleagues showed that fewer macrophages were present in the tissues surrounding MOM THAs than in the tissues surrounding MOPE THAs. Catelas and colleagues demonstrated that tissues surrounding failed MOM THAs with low to moderate quantities of metal particles could induce the production of potentially osteolytic cytokines. In addition to the potential for wear products to induce osteolysis through inflammatory response as observed surrounding conventional MOPE implants, a concern has emerged regarding the possibility of specific immunological responses. These immunological responses could be due to the release of metal ions that act as antigens and stimulate an allergic (hypersensitivity) reaction when they form organometallic complexes with proteins. Hypersensitivity reactions can be mediated by antibodies (type I, II, or III) or can be cellmediated (type IV, i.e., delayed type hypersensitivity). Type IV reactions to metal ions involve the activation and clonal expansion of metal-ion-specific major histocompatibility 
complex (MHC) class II restricted CD4+ T-lymphocytes by professional antigen-presenting cells (APCS). However, in addition to conventional peptide antigens, $\mathrm{T}$ cells can also react directly with metal ions. Regardless of the mechanisms, the consequences are that metal ions-sensitized CD4 ${ }^{+}$T-lymphocytes would elaborate cytokines, including interferon $\gamma$, that will attract and activate more macrophages, leading to the release of additional osteolytic cytokines and possibly causing a T-cell-mediated periprosthetic osteolysis.(Hallab 2001; Whittingham 2008) In a review study, Gawkrodger concluded that although early MOM displayed high incidences of hypersensitivity, no conclusion could be drawn about the new generation of MOM implants.(Gawkrodger 2003) Finally, it is still unclear if the loosening of the implants can be linked to an increased reactivity of the lymphocytes to metal particles or ions. When comparing tissues surrounding MOPE and MOM THAs, Campbell and colleagues showed more lymphocytes in the MOM THAs.(Campbell 2005) Although this infiltration of lymphocytes could be indicative of a delayed-type hypersensitivity response to the metal wear products and the development of a typical immunological response, Willert and colleagues also reported the presence of plasma cells, B-lymphocytes, and massive fibrin exudation, which are not characteristic of a type IV delayed-type hypersensitivity reaction. These authors described this reaction as an aseptic lymphocytedominated vasculitis-associated lesion (ALVAL) or as a lymphocyte-dominated immunologic (LYDIA) answer. Pandit et al. reported the presence of pseudotumors surrounding MOM surface replacements. These pseudotumors were characterized by an extensive necrosis of dense connective tissue, a focaly heavy macrophage and lymphocytic infiltration as well as the presence of plasma cells and eosinophils in some cases. The observed reaction was somewhat similar to ALVAL reaction reported by Willert et al. but with possibly a more diffuse lymphocyte infiltrate and the presence of extensive connective tissue necrosis.(Willert 2005) However, the specificity of such pseudotumors to MOM implants remains to be determined.(Pandit 2008) The potential adverse effects of MOM wear particles are mainly due to their local and systemic distribution and their capacity for releasing corrosion products, especially if they are in situ for several decades. Systemic dissemination of soluble and particulate corrosion products has been described, including the presence of metallic particles in the liver and spleen and raises questions about potential genotoxicity.(Urban 2000)

\subsubsection{Local and systemic effects}

Metal wear debris may cause DNA damage through the action of metal particles and/or through the action of metal ions by a chemical effect. Cobalt-chromium particles may exert a purely particulate effect that damages DNA by inducing free-radical formation in a way similar to the actions of nonmetallic particles. Alternatively, the cobalt-chromium particle might produce a chemical effect as a result of the release of cobalt and/or chromium ions. $\mathrm{Cr}^{3+}$ and $\mathrm{Cr}^{6+}$ cause DNA-strand breaks. $\mathrm{Co}^{2+}$ itself causes DNA-strand breaks, but in addition it inhibits DNA repair, therefore potentially augmenting the damage caused by chromium. Metal ions and wear debris may lead to genomic instability, similar to that induced by ionizing radiation, and this may increase the probability of DNA damage or mutation during normal cellular function.(Cobb 2006)

Temporary increases in the incidence of hematopoietic cancers at different follow-up points were observed in some cohorts. A study by Visuri et al. demonstrated a 3.77-fold increase in the rate of hematopoietic cancer following metal-on-metal total hip arthroplasty compared with the rate following metal-on-polyethylene total hip 
arthroplasty, but this difference was not significant.(Visuri 2006) Recent evidence from studies of patients who have undergone metal-on-metal hip resurfacing has shown raised circulating levels of cobalt and chromium ions to be associated with T-cell lymphopenia, with the lymphocyte counts of these patients differing from those of control subjects with a total hip arthroplasty that did not produce metal wear debris. The effect was consistent only for $\mathrm{CD}_{8}{ }^{+} \mathrm{T}$-cell lymphocytes.(Hart 2006, 2009)

Another concern regarding increased metal-ion levels following metal-on-metal total hip arthroplasty is that the ions may cross the placental barrier. Initially, it was thought that the placenta was an effective barrier to metal ions, but a subsequent study showed that the placenta actually exerts a modulatory effect on the rate of metal ion transfer. The transfer rate at low maternal metal-ion levels is higher than that at higher maternal levels. The transplacental transfer rate was in excess of $95 \%$ in the controls for both metals, but only $29 \%$ for chromium and $60 \%$ for cobalt in study patients, suggesting that the placenta exerts a modulatory effect on the rate of metal ion transfer.(Ziaee 2007) On the basis of these findings and the lack of comprehensive knowledge regarding the potential effects of metal ions on fetal development, metal-on-metal total hip arthroplasty probably should not be performed in women of child-bearing age until additional information is available.(Brodner 2004)

\subsubsection{Cancer risk}

$\mathrm{Co}$ and $\mathrm{Cr}$ wear particles have been shown to induce carcinoma in animal models, giving rise to the concern that such alloys could have the same effect in human tissues if present in sufficient amounts for a sufficient length of time. The first well-documented case of cancer associated with total joint replacement was in a patient who developed a malignant fibrous histiocytoma 3.5 years after a MOM THR performed in December 1969. There have been, at least, 24 additional cases reported in the English literature of malignancy occurring in association with a total hip or knee prostheses. Of the 25 reported cases of cancer following a total joint replacement, 21 involved sarcomas.(Gillespie 1988) The risk of cancer after MOM THR has been assessed specifically in only one epidemiological study. In that study, the relative risk of cancer was reported to be 0.95 , suggesting that there is no apparent increased risk of cancer development after MOM total hip arthroplasty. In addition, the risk of sarcoma after MOM THR was found to be 0.00 . However, the same study found the relative risk of hematopoietic cancer to be 1.59 following MOM THR and 3.77 for leukemia when MOM implants were compared with MOP implants. From an epidemiological perspective, these data are limited because of the small number of patients who underwent MOM THR. Furthermore, the majority of patients in these reports have less than 10 years of follow-up. The latency of known carcinogens, such as tobacco, asbestos, and ionizing radiation, is several decades. Longer follow-up of large patient groups is needed to better assess the risk of cancer with any implant system.(Tharani 2001) Since the goal of more wear resistant bearings is to reduce the need for reoperation, theoretical risks should be weighed against the risks of revision THR. In the Medicare population, the 90-day mortality following revision THR is $2.6 \%$, which is significantly and substantially higher than that of primary total hip and directly related to the revision procedure. Rigorous long-term studies are needed to assess the relative risk to benefit ratios for total hip bearings.

\subsection{Ceramic bearings}

Ceramic bearings, made of alumina, have demonstrated the lowest in vivo wear rates to date of any bearing combination. The same principles of friction and lubrication reported for 
MOM bearings apply to ceramic-on-ceramic (COC) bearings. However, ceramics have two important properties that make them an outstanding material regarding friction and wear. Ceramics are hydrophilic, permitting a better wettability of the surface. This ensures that the synovial fluid-film is uniformly distributed over the whole bearing surface area. Secondly, ceramic has a greater hardness than metal and can be polished to a much lower surface roughness. Although the better wettability results in a slightly thinner fluid-film than with MOM bearings it is compensated by the reduced size of the asperities on the surface. Overall, this results in a favorable higher $\lambda$ ratio and in a reduced coefficient of friction. This bearing combination is the most likely to achieve true fluid-film lubrication. However, because of the hardness of ceramics, the wear characteristics are sensitive to design, manufacturing, and implantation variables. Rapid wear has also been observed, generally associated with suboptimal positioning of the implants. COC bearings currently in clinical use are made of alumina. Developments in the production process (sintering) have improved the quality of the material. Modern alumina ceramics have a low porosity, low grain size, high density, and high purity. Thus, its hardness, fracture toughness, and burst strength increased. The alumina on alumina bearing is considered the standard ceramic on ceramic articulation.(D'Antonio 2009) Alumina ceramic bearings have been in clinical use for more than three decades, and significant basic science and clinical research support their use. The small amount of ceramic particulate debris that is generated has been noted to be much less biologically reactive than metal or polyethylene particles. Most important, the incidence of osteolysis associated with use of ceramic on ceramic bearings appears to be minimal or nonexistent. (Bierbaum 2002)

\subsubsection{Mechanical properties}

Alumina ceramics are classified as hard, stiff, and brittle materials. Because alumina ceramics are highly oxidized, they are biologically inert and resistant to further oxidation. The hardness of alumina creates a product with significant resistance to surface damage, and ceramics are much harder than other materials routinely used in orthopedic surgery. The hardness of alumina makes it very abrasive and wear resistant. In addition, the hardness of alumina increases its resistance to scratching, and it is much less likely to scratch than titanium or cobalt chromium alloys. In fact, the only material capable of scratching alumina is diamond. Clinically, this is important because alumina can resist third-body wear and is not scratched by retained cement particles or bone. Although alumina has poor bending characteristics, it is extremely strong in compression. This lack of bending strength has currently limited its use in total hip arthroplasty to the femoral head and cup liner. Because alumina is very stiff, it does not deform under high loads. Therefore very precise production techniques are needed in order to ensure proper fit of the head within the socket. Polyethylene will mold around a femoral head if there is an initial, small incongruity, which is not true with ceramics, and poor manufacturing can lead to high wear rates. The lack of ceramic deformation makes the contact areas between the head and socket smaller as compared with metal on polyethylene articulations. In order to maximize the contact surface area, clearance must be optimized. Alumina is more than 300 times stiffer than cancellous bone and almost 200 times stiffer than polymethlymethracrylate. Because of this significant modulus mismatch, cemented ceramic components have been found to be associated with higher cement fracture and loosening rates than all-polyethylene components. Alumina is very brittle and under compression will deform linearly until fracture. No plastic deformation occurs before fracture. By definition, its fracture toughness is considered to be 
its resistance to fracture. The initial flaws in the material determine the risk of ceramic fracture, and flaws are related to the purity and density of the ceramic. A combination of improvements, including improved processing with smaller grain sizes, fewer impurities, laser etching, and proof testing, have led to a lower incidence of material fracture. The burst strength of alumina components improved from 38 kilonewtons in 1977 to 98 kilonewtons in 1998. (Tateiwa 2008)

\subsubsection{Alumina and zirconia}

Medical-grade alumina is consolidated from a high-purity (>99\%) alumina powder through a hot isostatic pressing step. Modifications to the sintering process over the past three decades have resulted in increased density, hardness, and strength of medical-grade alumina. Modifications to the sintering also led to a decrease in the average grain size from $4.5 \mu \mathrm{m}$ to about $1.8 \mu \mathrm{m}$, with a narrower grain size distribution. The improved regulation of the grain size did not lead to any noticeable changes in the fracture toughness of alumina. While alumina remains the most widely used ceramic in the total hip, zirconia is also utilized as a replacement for alumina femoral heads, but only for applications where the counterface is UHMWPE. Zirconia is stronger than alumina; therefore it can withstand higher stresses, which may be induced when smaller and thinner implants are used. During deformation, pure zirconia undergoes a crystalline-phase transformation from monoclinic to tetragonal, with the former occupying more space. This transformation adversely affects the mechanical properties of pure zirconia, leading to internal stresses within the deformation zone ahead of propagating cracks. Zirconia can be stabilized with the addition of either magnesium oxide $(\mathrm{MgO})$ or yttrium oxide $\left(\mathrm{Y}_{2} \mathrm{O}_{3}\right)$ to avoid the detrimental effects of deformation-induced phase transformation.(De Aza 2002)

Alumina was the first ceramic to be widely used in hip arthroplasty, following its introduction in 1970. Aluminium oxide $\left(\mathrm{Al}_{2} \mathrm{O}_{3}\right)$ can be highly polished to produce a very low coefficient of friction and is also highly resistant to abrasion. It has a more stable structure than the more recently developed Zirconia so the properties are more predictable. Improved manufacturing techniques have resulted in smaller grain size and smoother finish helping to reduce the fracture risk that was encountered when using earlier generations. (D'Antonio 2009)

Zirconium oxide $\left(\mathrm{ZrO}_{2}\right)$ (Zirconia) was introduced in 1985 as an alternative to alumina. Its superior mechanical strength was attractive with a theoretical reduction in fracture risk. It also allowed smaller femoral head sizes whilst maintaining excellent wear characteristics when coupled with UHMWPE. Clinical trials have shown that UHMWPE wear when coupled with a zirconia femoral head is at least as good as that with a Co-Cr femoral head and in many cases is much better.(Affatato 2001) However Zirconia undergoes phase transformation. The strongest tetragonal phase is also the most unstable, so, for medical purposes, a stabilizer, Yttrium oxide, is used. In vivo studies have shown that phase transformation still occurs at the bearing surface secondary to temperature and pressure changes which transforms zirconia to the more stable monoclinic phase. This results in a 3\% increase in volume producing surface roughness. Hence it performs poorly when articulating against itself and can also result in accelerated polyethylene wear bearing on UHMWPE. As a result it has fallen out of favour.(Fernandez-Fairen 2007)

\subsubsection{Tribologic properties}

In vitro wear studies have proved that alumina on alumina is a very low friction couple, and wear is significantly reduced. The outstanding tribologic properties of the alumina 
articulation are due to its low surface roughness (secondary to small grain size), hardness, enhanced wettability, and fluid film lubrication. It has been shown that there are two wear phases during in vitro testing. The "run-in" phase is the first phase and involves the first million or so cycles. Volumetric wear rates for alumina against alumina bearings during this run-in phase measure 0.1 to $0.2 \mathrm{~mm}^{3}$ per million cycles. The second phase is called the "steady-state" phase. During this period, volumetric wear rates decrease to less than 0.02 $\mathrm{mm}^{3}$ per million cycles. Compared with metal on polyethylene couples, during both the run-in and steady-state phases, wear is reduced up to 5000 fold. Under certain clinical conditions, accelerated wear can occur with alumina on alumina couples. One phenomenon called "stripe wear" occurs when accelerated wear is present over a discrete area. Stripe wear may be associated with separation of the ball from the socket such as during the swing phase of gait or when the ball is levered out of the socket by impingement. In vitro testing under the conditions of separation of the femoral head from the socket leads to increased volumetric wear. It was noted that wear as high as $1.24 \mathrm{~mm}^{3}$ per million cycles could occur with separation and stripe wear results. A bimodal distribution of particle size was also noted in this study, with nanometer-sized particles (1 to $35 \mathrm{~nm})$ probably associated with polishing of the articulation and micrometer-sized particles $(0.05$ to $10 \mu \mathrm{m})$ that likely originated from stripe wear and transgranular fracture of the alumina ceramic. Numerous retrieval studies of ceramic bearings have been performed, and the results are interesting. One study examined retrieved alumina components associated with aseptic loosening of the socket at a mean of 11 years after implementation. Components were classified into three groups: (1) low wear with no visible signs of material loss; (2) stripe wear with a visible oblong worn area on the femoral head and a penetration rate below $10 \mu \mathrm{m} /$ year; and (3) severe wear with visible loss of material on both components and maximum penetration higher than $150 \mu \mathrm{m}$.(Fig.10 and 11) Evaluation of these 11 components revealed massive, severe wear on two devices. The remaining nine components had liner wear rates less than $15 \mu \mathrm{m} /$ year. The authors concluded that two different types of wear are associated with ceramic on ceramic couples-one that is limited and has negligible effect on long-term performance of the implant, and a second type that catastrophically leads to rapid destruction of the bearing surface. Published wear rates examining clinical performance of the alumina on alumina bearing surface have reported wear to range from $0.3 \mu \mathrm{m} /$ year to $5.0 \mathrm{~mm} /$ year. These variations may be related to implanted material and design issues or surgical and patient factors. However, it is important to note that most catastrophic wear

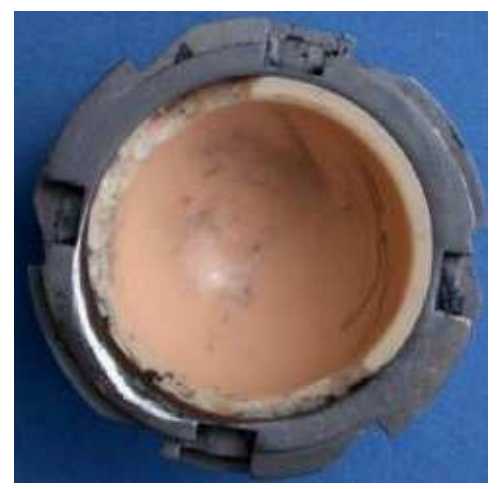

Fig. 10. Acetabular liner showing gross damage to the liner 
has been reported with products produced before 1990. In recent years, wear rates below 15 $\mu \mathrm{m} /$ year have been consistently reported. Many investigators believe that severe wear is related to clinically exceptional circumstances and that with properly implanted bearing surfaces, catastrophic wear is essentially nonexistent.(Capello 2008)

\subsubsection{Microseparation and wear particles}

Ceramic materials may have better biocompatibility than metal alloys, but the relative size, shape, number, reactivity, and local versus systemic distribution of the wear particles have not been fully determined. Hatton et al. investigated the tissues from ten noncemented COC THAs undergoing revision surgery. The tissues from the femoral and acetabular regions demonstrated the presence of intracellular particle agglomerates and mixed pathology, with areas that had no obvious pathology and areas that were relatively rich in macrophages, and over half of the tissues had $60 \%$ of necrosis. A bimodal size range of ceramic wear debris was observed, with particles as small as 5 to $90 \mathrm{~nm}$ (mean $24 \mathrm{~nm}$ ) and as large as 0.05 to 3.2 $\mu \mathrm{m}$ (mean $430 \mathrm{~nm}$ ). (Hatton 2002a) These two types of ceramic wear debris are generated by two different wear mechanisms in vivo, with very small wear particles being generated under normal articulating conditions and larger particles being generated under microseparation conditions. After total hip arthroplasty, the femoral head and the acetabular insert can separate up to $2 \mathrm{~mm}$ during the swing phase of a normal gait cycle. When a load is applied at heel strike in the stance phase, the femoral head moves vertically to relocate in the cup. With the geometry of a typical COC prosthesis, separations of only 2 $\mathrm{mm}$ will allow the femoral head to contact the rim acetabular liner, resulting in changes in the wear performance of the bearing couple (stripe wear). (Stewart 2001, 2003)

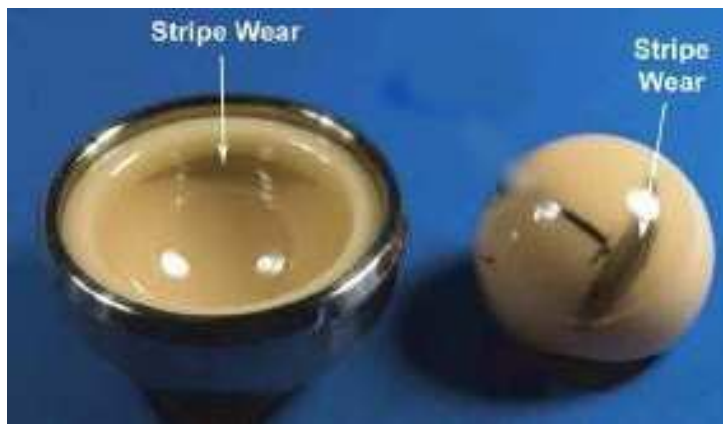

Fig. 11. A ceramic head and acetabular liner from a patient with "squeaking" hip with evidence of impingement and "stripe wear.

It is hoped that improvements in the manufacturing of ceramics and ceramic components will minimize or eliminate mechanical problems such as fracture and accelerated wear.

\subsubsection{Wear debris and tissue response}

It has been shown, in vitro and in vivo, that alumina wear debris is biologically inert and well tolerated. Alumina particles induce very little cellular response and formation of granulomatous tissue. The small nature of most alumina on alumina wear particles and the low volume of particles generated leads to a low level of bioactivity. Giant cells have not been observed in contact with alumina wear debris. Lerouge and colleagues compared 12 
periprosthetic membranes obtained during revision for aseptic component loosening with an alumina on alumina couple. These were compared with a series of membranes obtained from revisions of a metal on polyethylene bearing. In the alumina on alumina group, the cellular reaction, which was generally mild, was determined to be in response to the zirconia ceramic particles used in the cement as an opacifying agent. No cellular reaction to the alumina particles was noted.(Lerouge 1997) This contrasted with the significant cellular activity noted in the metal on polyethylene group with reaction to the polyethylene debris. Osteolysis associated with alumina on alumina total hip arthroplasty has been infrequently reported. In one study, when an implant made with large-grain-size ceramics, low density, and high porosity was used, large production of debris resulted and osteolysis occurred. Tissue obtained from failed hips with an alumina on alumina couple was shown to have significantly lower prostaglandin $\mathrm{E}_{2}\left(\mathrm{PGE}_{2}\right)$ levels compared with tissue obtained from hips with metal on polyethylene articulation. Both alumina and polyethylene debris stimulate cellular release of tumor necrosis factor (TNF)-a. However, polyethylene particles cause more release of TNF- $a$, and in fact the stimulation may be 8 to 10 times greater. Of importance, alumina particles induce macrophage apoptosis, which leads to decreased macrophage activity. This induced apoptosis explains the decreased levels of TNF- $\alpha$ associated with alumina and may also account for the paucity of ceramic-related osteolysis.

Ceramic debris may not be bio-inert as initially assumed, because osteolysis has been described in some patients with a COC bearing.(Nam 2007) Some studies describe inflammatory and cytotoxic reactions on the cellular level, but the relationship to material, size, and particle number remains uncertain. It seems that there is less inflammatory reaction compared to MOM or MOP bearings in well-functioning prostheses. In contrast with polyethylene or metallic particles, foreign body reactions are routinely observed. Ion toxicity is not an issue with ceramics.(Mehmood 2008)

\subsubsection{Ceramic-on-metal articulation}

In a simulator study, Firkins et al demonstrated superior wear for alumina ceramic-on-metal articulations. Femoral heads $28 \mathrm{~mm}$ in diameter made of medical-grade alumina were articulated against acetabular cups manufactured from medical-grade, high-carbon-wrought cobalt-chromium alloy. The implants were tested for 5 million cycles. Wear and surface analyses were performed every 1 million cycles. The results were compared with metal-onmetal articulations that used medical-grade, low-carbon, cobalt-chromium alloy femoral heads against medical-grade, high-carbon, cobalt-chromium alloy sockets. The metal-onmetal bearings initially showed high bedding-in and subsequently developed a steady-state volumetric wear rate of $1.23 \mathrm{~mm} 3$ per 1 million cycles. No bedding-in period was observed with the ceramic-on-metal, and a volumetric wear rate of $0.01 \mathrm{~mm} 3$ per 1 million cycles was reported. This represents more than a 100-fold decrease in wear with the ceramic-on-metal articulation. Surface analysis of the ceramic head showed no signs of wear or change in surface roughness. Metal particles from both articulations were of nanometer size (6 to 30 $\mathrm{nm}$ ). In addition, the ceramic-on-metal articulations produced slightly smaller particles although they were far fewer in number. (Firkins 2001) Clinical studies are currently under way to assess the performance of these bearings. The potential advantage of this novel ceramic-on-metal bearing is lower wear and the generation of significantly fewer metal particles compared with currently available metal-on-metal bearing surfaces. This bearing combination allows for the use of large femoral heads, similar to metal-on-metal bearings. With larger ceramic heads, the fracture risk is reduced, and an increased number of femoral 
head options are available to the surgeon. Potential disadvantages of this new bearing articulation include the risk of a ceramic femoral head fracture and its as-yet unknown clinical performance.(D'Antonio 2009)

\subsubsection{Ceramic advantages}

The potential advantages of using a ceramic on ceramic articulation for total hip arthroplasty can be quickly summarized as decreased wear and elimination of osteolysis. Osteolysis from wear debris is commonly viewed as the major obstacle blocking the development of a "lifetime" hip replacement. The need to eliminate wear and osteolysis has been magnified by the extension of indications for total hip arthroplasty to younger, more active, healthier patients with long life expectancies. The potential for decreased wear is derived from the tribologic properties inherent to alumina. Alumina can be highly polished. Alumina bearings are also very hard, and this characteristic increases their resistance to scratching. The hardness minimizes third-body wear from entrapped bone, polymethylmethacrylate, or metal debris derived from surgical instruments or component fretting. Alumina has ionic properties and therefore, in combination with body fluids, has better wettability than chrome cobalt. The fluid film that develops on ceramic surfaces decreases frictional drag and adhesive wear. Wear rates for modern ceramic on ceramic articulations have been shown to be as low as $4 \mu \mathrm{m} /$ year. This low wear rate coupled with less alumina bioreactivity minimizes the likelihood of osteolysis. With currently used implant designs, osteolysis has not been reported with follow-up as long as 18.5 years.

\subsubsection{Ceramic concerns}

One significant drawback of ceramic materials is their inherently lower strength and toughness under tension and bending, which are the loading modes that favor the initiation and propagation of cracks. One such adverse loading arises from the mismatch at the taper junction between the metal stem and ceramic head. To prevent this from leading to early fracture, the tolerances at the taper are matched and may be manufacturer-specific. The surgical placement of the components may also predispose the ceramic components to high stresses and initiate fracture. For instance, a third body (bone cement or bone fragment) left in the taper interface or impingement of the femoral component on the rim of the ceramic acetabular liner secondary to malpositioning of the components could also initiate ceramic fracture.(Barrack 2004)

\subsubsection{Fracture (Fig.12)}

Improved material processing, smaller grain sizes, fewer impurities, laser etching, and proof testing have greatly diminished the risk of catastrophic in vivo fracture. The risk of ceramic fracture is estimated to have decreased nearly 100-fold in the last two decades. In 1990 the incidence of fracture was approximately $0.8 \%$, and today is likely between $0.004 \%$ and $0.010 \%$. Nonetheless, this complication is devastating and still occurs.(Min 2007) Even with proof testing, it is unlikely that failure by fracture will be eliminated. Although theoretically proof testing eliminates weaker components, flawed products that are likely to fail are not always eliminated. Proof testing theoretically is designed to be stringent enough to remove components with manufacturing flaws that are likely to clinically fail. However, the test must be nondestructive and not cause damage to the tested part. No proof test currently available is $100 \%$ effective. So, it must be remembered that production errors can and still do occur. In 1998 a manufacturing change resulted in a high fracture rate of ceramic balls. 
About one in three components clinically failed, and this was despite negative proof testing of all fractured devices. The production of ceramics is far more demanding than the manufacture of metal and polyethylene components, and the incidence of catastrophic failure for ceramics will always be higher than with other materials. Ceramic component fracture may occur secondary to poor surgical technique. Improper component insertion predisposes the implant to fracture. Impaction of the femoral head on the trunnion should be performed only after ensuring it is concentrically placed. Placing the head nonconcentrically on the trunnion or not cleaning and drying it properly leads to stress concentrations in the femoral head.(Poggie 2007) In addition, placing a ceramic head on a damaged trunnion also leads to stress concentration and a significantly reduced burst strength with the potential for fracture.(Anwar 2009) It is also possible to nonconcentrically place the ceramic liner in the metal acetabular shell. However, the adverse effects and longterm consequences of this error have not been reported. Ceramic component fracture is a double-edged sword. After fracture the patient is confronted with immediate debilitating pain and the need for emergency revision surgery. However, secondarily, revision of a fractured ceramic component carries the risk of a less than optimal outcome. Because of trunnion damage, revision with a ceramic head is usually not possible. Ceramic fracture debris embedded in the soft tissue can cause third-body wear and premature failure owing to accelerated wear if a metal and polyethylene articulation is used for the revision. During revision of fractured ceramic components, it is now recommended to perform meticulous synovectomy and debridement to remove as much fracture debris as possible and revise the femoral head with a metallic alloy, such as $\mathrm{CoCr}$, to avoid further catastrophic failure. Another option on the femoral side is to revise the femoral stem and use a ceramic femoral head.(Ha 2007; D'Antonio 2009)

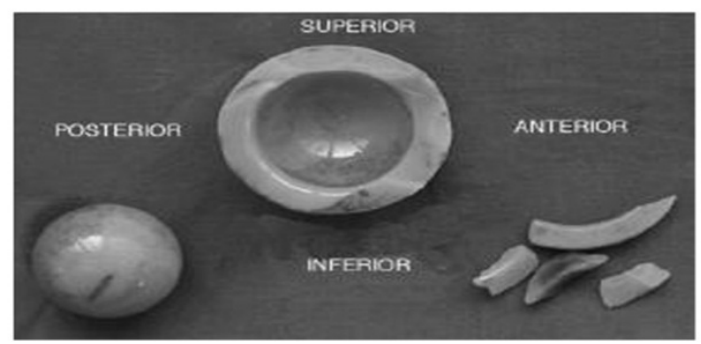

A

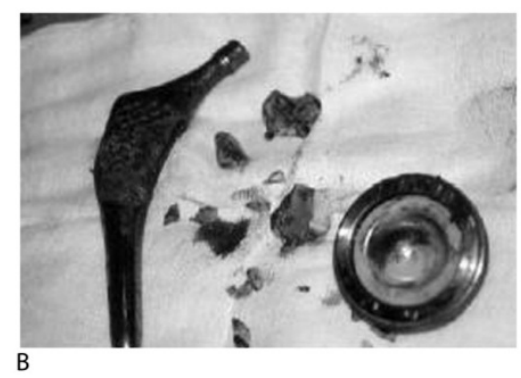

B

Fig. 12. Retrieved Ceramic-on-Ceramic implants showing fractured acetabular liner (A) and femoral head (B)

\subsubsection{Stripe wear}

Separation of the ball from the socket in patients with a hip arthroplasty may occur during the swing phase of gait or with impingement of the trunnion on the acetabular rim levering the ball from the socket. When this separation occurs, the contact area of the femoral head on the acetabular liner becomes small and stripe wear can result.(Fig.15) Stripe wear is concerning because volumetric wear associated with this phenomenon is high. In one study, stripe wear produced volumetric wear that averaged $1.24 \mathrm{~mm}^{3}$ per million cycles. Equally concerning, a bimodal array of nanometer- and micrometer-sized particles was created with an enhanced profile of bioreactivity. Separation of the ceramic on ceramic hip articulation is 
most likely to occur in individuals with tissue laxity or excellent range of motion. Also, patients with vigorous lifestyles and those who perform activities that require placing the hip through a provocative range of motion may be prone to impingement and stripe wear.(Walter 2004) For patients with these risk factors, other articulation choices should be considered. Of course, malpositioned components, as with any articulation, increase the risk of impingement. (Park 2006)

\subsubsection{Motion-related noise}

Hard on hard bearings can produce noise that can be disconcerting and annoying enough that revision surgery is requested by the patient. Specifically, with alumina on alumina bearings patients may describe this noise as "squeaking."(Keurentjes 2008) Ranawat and his colleagues reported that 10 of 159 ceramic on ceramic articulations squeaked and that the phenomenon was self-reported by the patient. The squeaking usually occurred in midrange of motion and was generally considered a significant issue of concern for the patient. In a study on patients with COC THAs who had squeaking, ideal cup position was described as 25 degrees \pm 10 degrees of anteversion and 45 degrees \pm 10 degrees of inclination. Clearly, these data suggest that squeaking is related to cup position.(Ranawat 2007; Walter 2007) However, conversely, Parvizi and associates reviewed the incidence of squeaking after 1056 ceramic on ceramic total hip arthroplasties. Thirty-three patients reported squeaking. The patients who had a noisy hip were matched against a control group, and cup position for both groups was carefully determined using computed tomography. There was no significant difference in acetabular position between the two groups.(Restrepo 2008) Retrieval of revised noisy ceramic bearings has so far been inconclusive. Noisy retrieved bearings frequently have a small zone of stripe wear and metallic staining, possibly associated with the head subluxing and making contact with the protective metal rim around the ceramic or the acetabular shell. (Taylor 2007) The management of squeaking begins with informed consent. The occurrence of noise in an otherwise well-functioning arthroplasty is very disconcerting to the patient. The patient's perception that the articulation is malfunctioning can prompt litigation which could involve the surgeon. Reassurance is appropriate because there is no evidence at this time that the noise reflects that the articulation is deteriorating. Certainly the patient should avoid provocative activities because this recommendation seems logical. There is empirical evidence that squeaking is related to a "dry" joint, and oral hydration or injection of viscosupplements can alleviate the noise. If a patient presents with a squeaking ceramic hip, first ceramic breakage must be excluded with a computed tomography scan because a broken ceramic insert can cause squeaking and it may not be obvious on plain radiographs. The computed tomography scan also provides an accurate measure of the acetabular component anteversion. If the noise itself is infrequent, and occurs only with a specific activity, then revision surgery may not be necessary. If the noise itself is troubling, if there is gross component malposition, if there is impingement, or if the patient has symptoms of pain, revision surgery should be recommended. The cup and stem need to be carefully inspected to be sure they are correctly positioned and well fixed. Because the trunnion may be damaged, revision to a metal ball and polyethylene socket is best, as metal on metal articulations have also been reported to occasionally make noise. Using a metal on metal articulation, if it also squeaks, would not likely be acceptable to the patient.(Walter 2008) 


\subsubsection{Other ceramic concerns}

The number of femoral head size and neck length options available to the surgeon when using ceramic articulations is limited. In general, for each acetabular component there is only one size liner diameter and paired head girth available. In addition, collared heads are not manufactured, and this significantly reduces the neck length options available. Also, offset and lipped acetabular liners are not produced because they would lead to impingement and chipping. These limitations have caused some experts to speculate that instability and dislocations will be more common when ceramic on ceramic bearings are used. However, clinically this has not proved to be a problem. Impingement of the femoral component trunnion on the ceramic liner edge is also an area of concern. This impingement can lead to chipping of the ceramic liner or notching of the femoral component. A protective metal rim has been added to the ceramic shell liner. Of course, impingement can still occur; however, this protective rim does seem to prevent ceramic damage. Nonetheless, impingement with the protective rim may still cause femoral neck notching and generate metallic debris. The consequences of this type of impingement are unknown; however, the generation of metallosis may theoretically contribute to the problem of noise and squeaking.

\subsubsection{Delta ceramic-on-alumina ceramic articulation in primary THA}

The newest generation of Ceramic-on-Ceramic bearings incorporate nanosized, yttriastabilized tetragonal zirconia particles producing an alumina matrix composite. This new bearing surface provided high survivorship.

The newest generation of ceramics, incorporating zirconia into the alumina matrix (marketed as Biolox delta by CeramTec, Plochingen, Germany). Nanosized, yttria-stabilized tetragonal zirconia particles improve the mechanical properties by preventing the initiation and propagation of cracks. Oxide additives produce platelet-like crystals that dissipate energy by deflecting cracks. The addition of chromium oxide further increases the strength and toughness by composite hardening. The final product is a mixture of roughly $75 \%$ aluminum oxide, $25 \%$ zirconia, and less than $1 \%$ chromium oxide and strontium oxide. The result is a composite ceramic with improved mechanical properties and reduced wear as shown on scanning electron microscopy (SEM) and a predicted longer lifespan. These improved mechanical properties reduce overall wear rates of $28-\mathrm{mm}$ heads in a hip simulator wear test from $1.84 \mathrm{~mm}^{3}$ per million cycle to $0.16 \mathrm{~mm}^{3}$ per million cycle, comparing alumina-on-alumina versus alumina matrix composite-on-alumina matrix composite, respectively. (Lombardi 2010; Hamilton 2010)

\subsubsection{Other advances}

There are some laboratory and very short-term clinical data to support the use of ceramic femoral heads articulating with metal acetabular components. These appear to produce less stripe wear with edge loading than metal-on-metal implants. It also appears that there is a reduction in metal ions in the patients at 6 months. Though there have been reports of early catastrophic failure if this pairing is reversed and a metal head is articulated with a ceramic acetabular component.

\subsubsection{Fabrication and testing of silicon nitride bearings in total hip arthroplasty}

A concern with ceramic femoral heads is brittle catastrophic failure in vivo. Improvements in the quality and manufacturing of $\mathrm{Al}_{2} \mathrm{O}_{3}$ have not eliminated this risk. In the past, zirconia 
femoral heads were introduced as a stronger alternative to $\mathrm{Al}_{2} \mathrm{O}_{3}$, but zirconia can undergo phase transformation and weakening, leading to unpredictable outcomes in vivo. Another strategy to reduce polyethylene wear is to modify the surface of zirconium alloy femoral heads. This avoids brittle failure, but the wear reduction, surface hardness, and scratch resistance of oxidized zirconium are less favorable than $\mathrm{Al}_{2} \mathrm{O}_{3}$, and oxidized zirconium cannot be used in ceramic-ceramic articulations. An alternative ceramic material was fabricated from silicon nitride $\left(\mathrm{Si}_{3} \mathrm{~N}_{4}\right)$ powder. Mechanical testing showed high-flexural strength, fracture toughness, Weibull modulus, and resistance to hydrothermal degradation. The $\mathrm{Si}_{3} \mathrm{~N}_{4}$ cups produced low wear rates when tested with either $\mathrm{CoCr}$ or $\mathrm{Si}_{3} \mathrm{~N}_{4}$ femoral heads. The $\mathrm{Si}_{3} \mathrm{~N}_{4}$ showed a near $100 \%$ theoretical material density $\left(3.20 \mathrm{~g} / \mathrm{cm}^{3}\right)$ and a uniform microstructure of fine, elongated grains (average grain width, $1.5 \mu \mathrm{m}$ ). The flexural strength was $923 \pm 70 \mathrm{MPa}$, with a Weibull modulus of 19 , and a fracture toughness of $10 \pm 1 \mathrm{MPa} \cdot \mathrm{m}^{1 / 2}$.

The $\mathrm{Si}_{3} \mathrm{~N}_{4}-\mathrm{Si}_{3} \mathrm{~N}_{4}$ and $\mathrm{Si}_{3} \mathrm{~N}_{4}-\mathrm{CoCr}$ bearings produced wear volumes of $0.2 \mathrm{~mm}^{3}$ and $0.18 \mathrm{~mm}^{3}$, respectively, at 1 million cycles. These data were extrapolated to 10 million cycle wear because previous hip simulator studies have shown that hard-on-hard bearing wear follows a characteristic biphasic pattern with higher wear in the early, run-in period to about 0.5 million cycles, followed by a lower, steady state linear wear pattern that can be extrapolated to predict long-term wear. A consistent wear rate, that is, the gradient of the linear regression trend is considered more significant than the actual magnitude of wear at any point in time. Accordingly, we calculated the volumetric wear rate from the slope of the linear regression curve during steady state wear; thus, at 10 million cycles, the predicted wear for $\mathrm{Si}_{3} \mathrm{~N}_{4}-\mathrm{Si}_{3} \mathrm{~N}_{4}$ and $\mathrm{Si}_{3} \mathrm{~N}_{4}$-CoCr bearings was calculated to be $0.65 \mathrm{~mm}^{3}$ and $0.47 \mathrm{~mm}^{3}$, respectively. For THA bearings of the same diameter, the clinical wear volume of $\mathrm{CoCr}$ polyethylene THA is $62 \mathrm{~mm}^{3}$, and the experimental wear volumes of $\mathrm{CoCr}-\mathrm{CoCr}$ and $\mathrm{Al}_{2} \mathrm{O}_{3}-$ $\mathrm{Al}_{2} \mathrm{O}_{3}$ measured in other hip simulators are $6.5 \mathrm{~mm}^{3}$ and between 0.35 and $0.6 \mathrm{~mm}^{3}$, respectively. After wear testing, $\mathrm{Si}_{3} \mathrm{~N}_{4}$ surfaces remained smooth and glossy, without scratches or damage.

Modern $\mathrm{Si}_{3} \mathrm{~N}_{4}$ is used in aerospace bearings and in high-temperature applications such as diesel engines. The $\mathrm{Si}_{3} \mathrm{~N}_{4}-\mathrm{Si}_{3} \mathrm{~N}_{4}$ pairings have a friction coefficient of 0.001 compared to 0.08 for $\mathrm{Al}_{2} \mathrm{O}_{3}-\mathrm{Al}_{2} \mathrm{O}_{3}$. The $\mathrm{CoCr}-\mathrm{Si}_{3} \mathrm{~N}_{4}$ articulations produced low wear rates in this study because of the favorable material hardness, tensile strength, fracture toughness, and lubricity of $\mathrm{Si}_{3} \mathrm{~N}_{4}$ and the close match in elastic modulii between the bearing materials. Because $\mathrm{Si}_{3} \mathrm{~N}_{4}$ has a higher fracture toughness than $\mathrm{Al}_{2} \mathrm{O}_{3}$, it is more resistant to the initiation and propagation of microcracks that can lead to brittle failure. The fracture toughness and strength of $\mathrm{Si}_{3} \mathrm{~N}_{4}$ also result in superior damage resistance, that is, the ability of the material to retain strength after contact damage. Practically, this means that $\mathrm{Si}_{3} \mathrm{~N}_{4}$ can withstand high loads even if contact stress-related damage were to occur.

It has been postulated that hard bearings in THA could produce a greater number of smaller-sized wear particles, thereby increasing the exposed surface area of bioreactivity in vivo. Wear particles were characterized in this study, and those findings have been reported separately. Because wear between dissimilar mating surfaces originates from the softer, sacrificial material, $\mathrm{Si}_{3} \mathrm{~N}_{4}-\mathrm{CoCr}$ couplings should generate predominantly $\mathrm{CoCr}$ metal particles. Assuming so, the volume of metal wear from $\mathrm{Si}_{3} \mathrm{~N}_{4}-\mathrm{CoCr}$ calculated in this study was an order of magnitude lower than the wear of retrieved $\mathrm{CoCr}-\mathrm{CoCr}$ THA bearings. Furthermore, particle size analysis has shown a mean equivalent circular diameter (ECD) of $\mathrm{Si}_{3} \mathrm{~N}_{4}$ wear particles of $0.7 \pm 0.6 \mu \mathrm{m}$ in this study. This figure is comparable to ultrahigh- 
molecular-weight polyethylene (mean ECD, approximately $0.4 \pm 0.2 \mu \mathrm{m}$ ) and $\mathrm{Al}_{2} \mathrm{O}_{3}$ (mean ECD, approximately $0.4 \pm 0.4 \mu \mathrm{m}$ ) particles obtained from clinical retrievals. Furthermore, the morphology of $\mathrm{Si}_{3} \mathrm{~N}_{4}$ wear particles in this study was similar to $\mathrm{Al}_{2} \mathrm{O}_{3}$ particles produced under similar testing conditions. For the same size and concentration, ceramic particles may be less bioreactive than those of polyethylene. Previous biocompatibility tests have shown that $\mathrm{Si}_{3} \mathrm{~N}_{4}$ does not elicit adverse in vivo reactions; specifically, exposure to $\mathrm{Si}_{3} \mathrm{~N}_{4}$ does not produce acute or chronic toxicity, mutagenicity, allergenicity, carcinogenicity, or localized tissue toxicity.(Bal 2009)

\section{New horizons in bearing surfaces}

Metal on polyethylene, metal on metal, and ceramic on ceramic bearings will continue as the dominant bearing materials for total hip arthroplasty because of their excellent track record, resistance to damage, and ease of manufacture and use. Clinically, most bearing combinations consist of cobalt-chromium $(\mathrm{CoCr})$ alloy or ceramic femoral heads articulating against highly cross-linked ultra-high-molecular weight polyethylene acetabular inserts. Alumina on alumina ceramic and $\mathrm{CoCr}$ on $\mathrm{CoCr}$ articulations are used in younger and more active patients. Each bearing combination has advantages and potential disadvantages. Second-generation HXPE materials are now available. Ceramic composite materials with high fracture toughness are under clinical investigation, as are ceramic on $\mathrm{CoCr}$ articulations. Outside this main area of bearing development, research efforts have been under way for some 20 years with two quite different classes of materials. The two classes of materials are carbon fiber polymeric composites and elastomeric materials.

\subsection{Carbon fiber polymeric composites}

Carbon fiber ultra-high-molecular weight polyethylene (UHMWPE) composites were introduced in the early 1980s as tibial bearings.(Fruh 1998) However, the clinical performance was poor with many revisions. Load can be transferred only by compressive forces between the fibers and matrix, resulting in an inefficient reinforcement. Carbon fiberreinforced epoxy composites (CFRP) were introduced in Europe in the late 1980s as acetabular components with alumina femoral heads. Simulator studies reported a wear rate of 1 to $3 \mu \mathrm{m}$ per million cycles. Canine hips with CFRP cups were implanted in six dogs for up to 5.5 years with no adverse consequences. A clinical study began in 1989, and 101 patients received cups of CFRP. In cases of revision, there were few particles in the tissue and the biologic response was benign. The wear of retrieved components was 6.1 to 6.3 $\mu \mathrm{m} /$ year. These wear rates are comparable to those seen with HXPE materials. Epoxy resins are thermoset materials and cannot be shaped other than by machining once they have "set" via chemical reaction. Many thermoplastic composites can be shaped by combinations of heat and pressure and "set" thereafter. Polyether ether ketone (PEEK) is a thermoplastic high performance polymer that is attractive as a composite matrix owing to its strength, toughness, stability, and biocompatibility.(Wang 1999)

\subsection{Compliant bearings}

There has long been interest in developing bearing materials that exhibit friction and wear behavior similar to that of articular cartilage. Cartilage is an example of a compliant bearing that has a low modulus but is capable of large deformation without failure. The friction coefficient between cartilage surfaces in a synovial joint is less than 0.01 . This low friction is 
achieved via three lubrication mechanisms: elastohydrodynamic (EHD) lubrication, $\mu$ EHD, and squeeze-film lubrication. Elastic deformation of the articular surfaces under load assists in allowing joint fluid to separate the surfaces, avoiding solid-solid contact with consequent wear. $\mu E H D$ has a similar effect confined to the surface asperities (roughness) of the cartilage. EHD and $\mu \mathrm{EHD}$ predominate during the stance phase of walking, when pressure is generated in the synovial fluid by an entraining motion between the joint surfaces. Squeeze-film action predominates during heel strike, as the two cartilage surfaces move toward each other, squeezing the joint fluid from between the surfaces. Deformation of the articular cartilage assists in retention of the synovial fluid film. Healthy cartilage has a thickness of 2 to $4 \mathrm{~mm}$ at the femoral head and acetabulum. The modulus of elasticity is 10 to $50 \mathrm{MPa}$ with a Poisson ratio of 0.42 to 0.47 . The surface roughness is about $2 \mu \mathrm{m}$. Polyurethanes are synthetic polymers having properties comparable to those of articular cartilage. There have been extensive studies to determine the suitability of polyurethanes as bearing surfaces. The intent is to have a bearing in which the surfaces are separated by the pressure developed in the joint fluid as well as by the deformation of the articular surfaces, resulting in low friction and low wear. This is a different approach from that of using UHMWPE, CoCr, or alumina bearings that operate under mixed lubrication conditions with higher friction and a degree of solid to solid contact.(Scholes 2000)

\section{References}

Affatato S, et al. (2005). Wear behaviour of crosslinked PE assessed in vitro under severe conditions. Biomaterials, 26, 2005: 3259-3267

Affatato S, et al. (2008). Predictive role of the lambda ratio in the evaluation of metal-onmetal total hip replacement. Proc Inst Mech Eng, 222, 2008: 617-628

Antoniou J, et al. (2008). Metal ion levels in the blood of patients after hip resurfacing: a comparison between twenty-eight and thirty-six-millimeter-head metal-on-metal prostheses.J Bone Joint Surg Am, 90 (Suppl 3), 2008: 142-148

Anwar I, et al. (2009). Delayed catastrophic failure of a ceramic head in hybrid total hip arthroplasty. J Arthroplasty, 24, 2009: 158-162

Archibeck MJ, et al. (2000). The basic science of periprosthetic osteolysis. J Bone Joint Surg Am, 82, 2000: 1478-1489

Atienza C Jr, Maloney WJ. (2008). Highly cross-linked polyethylene bearing surfaces in total hip arthroplasty. J Surg Orthop Adv, 17, 2008: 27-33

Bagchi D, et al. (2002). Cytotoxicity and oxidative mechanisms of different forms of chromium. Toxicology. 180, 2002: 5-22.

Baker D, et al. (2003). The effects of degree of crosslinking on the fatigue crack initiation and propagation resistance of orthopedic grade polyethylene. J Biomed Mater Res, 66, 2003: 146

Bal BS, et al. (2009). Fabrication and Testing of Silicon Nitride Bearings in Total Hip Arthroplasty. J Arthroplasty, 24, 1, 2009: 110-116

Barnes CL, et al. (2008). Wear Performance of Large-Diameter Differential-Hardness Hip Bearings. J Arthroplasty, 23(6), Suppl. 1, 2008: 56-60

Barrack RL, et al. (2004). Concerns about ceramics in THA. Clin Orthop Relat Res, 429, 2004: 73-79 
Beaul'e PE, et al. (2001). Osteolysis in a cementless, second generation metal-on-metal hip replacement. Clin Orthop Relat Res, 386, 2001: 159-165

Bhamra MS, Case CP. (2006). Biological effects of metal-on-metal hip replacements. Proc Inst Mech Eng, 220, 2006: 379-84

Bierbaum BE, et al. (2002). Ceramic-on-ceramic bearings in total hip arthroplasty. Clin Orthop Relat Res, 405, 2002: 158-63

Bitsch RG, et al. (2007). Ion Production and Excretion in a Patient with a Metal-on-Metal Bearing Hip Prosthesis. A Case Report. J Bone Joint Surg Am, 89, 2007: 2758-2763

Bozic KJ, et al. (2009). The Epidemiology of Bearing Surface Usage in Total Hip Arthroplasty in the United States. J Bone Joint Surg Am, 91, 2009: 1614-1620

Bradford L, et al. (2004). Early failure due to osteolysis in highly cross-linked ultrahigh molecular weight polyethylene: a case report. J Bone Joint Surg Am, 86A, 2004: 1051

Brodner W, et al. (2003). Serum cobalt levels after metal-on-metal total hip arthroplasty. J Bone Joint Surg Am, 85, 2003: 2168-2173

Brodner W, et al. (2004). Does the placenta inhibit the passage of chromium and cobalt after metal-on-metal total hip arthroplasty? J Arthroplasty, 19 (Suppl), 2004: 102-106

Buford A, Goswami T. (2004). Review of wear mechanisms in hip implants. Mat Des, 25, 2004: 385-393

Campbell PA, et al. (2005). An unusual lymphocytic perivascular infiltration in tissues around contemporary metal-on-metal joint replacements. J Bone Joint Surg Am, 87, 2005: 18-27

Capello WN, et al. (2008). Ceramic-on-ceramic total hip arthroplasty: update. J Arthroplasty, 23, 2008: 39-43

Catelas I, et al. (2006). Wear particles from metal-on-metal total hip replacements: effects of implant design and implantation time. Proc Inst Mech Eng, 220, 2006: 195-208

Chan FW, et al. (1996). Engineering issues and wear performance of metal on metal hip implants. Clin Orthop Relat Res, 333, 1996: 96-107

Chan FW, et al. (1999). Wear and lubrication of metal-on-metal hip implants. Clin Orthop Relat Res, 369, 1999: 10-24

Clarke IC, Gustafson A. (2000). Clinical and hip simulator comparisons of ceramic-onpolyethylene and metal-on-polyethylene wear. Clin Orthop Relat Res, 379, 2000: 3440

Cobb AG, Schmalzreid TP. (2006). The clinical significance of metal ion release from cobaltchromium metal-on-metal hip joint arthroplasty. Proc Inst Mech Eng, 220, 2006: 385398

D'Antonio J, et al. (2009). Ceramic Materials as Bearing Surfaces for THA. J Am Acad Orthop Surg, 17, 2009: 63-68

Daniel J, et al. (2006). The effect of the diameter of metal-on-metal bearings on systemic exposure to cobalt and chromium. J Bone Joint Surg Br, 88, 2006: 443-448

Daniel J, et al. (2007). The validity of serum as a surrogate measure of systemic metal ion exposure in hip arthroplasty. J Bone Joint Surg [Br], 89B, 2007 : 736-741

De Aza AH, et al. (2002). Crack growth resistance of alumina, zirconia and zirconia toughened alumina ceramics for joint prostheses. Biomaterials, 23, 2002: 937-945 
Digas G, et al. (2003). Increase in early polyethylene wear after sterilization with ethylene oxide: radiostereometric analyses of 201 total hips. Acta Orthop Scand, 74, 2003: 531541

Digas G, et al. (2007). 5-year experience of highly cross-linked polyethylene in cemented and uncemented sockets. Two randomized studies using radiostereometric analysis. Acta Orthop, 78(6), 2007: 746-754

Dowson D, et al. (2004). A hip joint simulator study of the performance of metal-on-metal joints. Part II: design. J Arthroplasty, 19 (Suppl 3), 2004: 124-130

Dowson D, Jin ZM. (2006). Metal-on-metal hip joint tribology. Proc Inst Mech Eng, 220, 2006: 107-118

Dumbleton JH, et al. (2006). The basis for a second-generation highly cross-linked UHMWPE. Clin Orthop, 453, 2006: 265-271

Engh CA, et al. (2009). Metal Ion Levels After Metal-on-Metal Total Hip Arthroplasty: A Randomized Trial. Clin Orthop Relat Res, 467, 2009: 101-111

Fernandez-Fairen $M$, et al.( 2007). Aging of Retrieved Zirconia Femoral Heads. Clin Orthop Relat Res, 462, 2007, 122-129

Firkins PJ, et al. (2001) A novel low wearing differential hardness, ceramic-on-metal hip joint prosthesis. J Biomech, 34, 2001: 1291-1298

Fisher J, et al. (2004). Wear of surface engineered metal-on-metal hip prostheses. J Mater Sci Mater Med, 15, 2004: 225-235

Fruh HJ, Willmann G. (1998). Tribological investigations of the wear couple alumina-CFRP for total hip replacement. Biomaterials, 19, 1998: 1145-1150

Garvin KL, et al. (2009). Wear Analysis in THA Utilizing Oxidized Zirconium and Crosslinked Polyethylene. Clin Orthop Relat Res, 467, 2009: 141-145

Gawkrodger DJ. (2003) Metal sensitivities and orthopedic implants revisited: the potential of metal allergy with the new metal-on-metal joint prostheses. Br J Dermatol, 148, 2003: 1089

Geerdink CH, et al. (2009). Cross-linked Compared with Historical Polyethylene in THA An 8-year Clinical Study. Clin Orthop Relat Res, 476, 2009: 979-984

Gillespie WJ, et al. (1988). The incidence of cancer following total hip replacement. J Bone Joint Surg Br, 70, 1988: 539-542

Glaser D, et al. (2008). Clicking and Squeaking: In Vivo Correlation of Sound and Separation for Different Bearing Surfaces. J Bone Joint Surg Am, 90, 2008: 112-120

Greenbaum ES, et al. (2004). Effect of lipid absorption on wear and compressive properties of unirradiated and highly crosslinked UHMWPE: an in vitro experimental model. Biomaterials, 25, 2004: 4479-4484

Gul RM, et al. (2003). Effect of consolidation on adhesive and abrasive wear of UHMWPE. Biomaterials, 24, 2003: 3193-3199

Ha YC, et al. (2007). Ceramic liner fracture after cementless alumina-on-alumina total hip arthroplasty. Clin Orthop Relat Res, 458, 2007: 106-110

Hallab N, et al. (2001). Metal sensitivity in patients with orthopaedic implants. J Bone Joint Surg Am, 83, 2001: 428-36

Hamilton WG, et al. (2010). THA with Delta Ceramic on Ceramic; Results of a Multicenter Investigational Device Exemption Trial. Clin Orthop Relat Res, 468, 2010: 358-366 
Hannouche D, et al. (2003). Fractures of ceramic bearings: history and present status. Clin Orthop Relat Res, 417, 2003: 19-26

Hannouche D, et al. (2005). Ceramics in total hip replacement. Clin Orthop Relat Res, 430, 2005: $62-71$

Harris WH. (2001). Wear and periprosthetic osteolysis: the problem. Clin Orthop Relat Res, 393, 2001: 66-70

Hart AJ, et al. (2006). The association between metal ions from hip resurfacing and reduced T-cell counts. J Bone Joint Surg Br, 88, 2006: 449-454

Hart AJ, et al. (2009). Circulating levels of cobalt and chromium from metal-on-metal hip replacement are associated with $\mathrm{CD}_{8} \mathrm{~T}$-cell lymphopenia. J Bone Joint Surg Br, 91, 2009: 835-842

Hatton A, et al. (2002a). Alumina-alumina artificial hip joints: Part I. A histological analysis and characterisation of wear debris by laser capture microdissection of tissues retrieved at revision. Biomaterials, 23, 2002: 3429

Hatton A, et al. (2002b). Alumina-alumina artificial hip joints. Part II: characterisation of the wear debris from in vitro hip joint simulations. Biomaterials, 23, 2002: 3441-3448

Hern'andez-Rodr'iguez MAL, et al. (2005). Wear of cast metal-metal pairs for total replacement hip prostheses. Wear, 259, 2005: 958-963

Howcroft D, et al. (2008). Bearing surfaces in the young patient: out with the old and in with the new? Current Orthopaedics, 22, 2008: 177-184

Huber M, et al. (2010). Postmortem Study of Femoral Osteolysis Associated with Metal-onMetal Articulation in Total Hip Replacement; An Analysis of Nine Cases. J Bone Joint Surg Am, 92, 2010: 1720-1731

Isaac GH, et al. (2006). Metal-on-metal bearings surfaces: materials, manufacture, design, optimization, and alternatives. Proc Inst Mech Eng, 220, 2006: 119-133

Jacobs JJ, et al. (1998). Metal release in patients who have had a primary total hip arthroplasty. A prospective, controlled, longitudinal study. J Bone Joint Surg Am, 80, 1998: 1447-1458

Jacobs JJ, et al. (2006). Wear Particles. J Bone Joint Surg Am, 88, 2006: 99-102

Jarrett CA, et al. (2009). The squeaking hip: an underreported phenomenon of ceramic-onceramic total hip arthroplasty. J Bone Joint Surg Am, 91, 2009: 1344-1349

Keurentjes JC, et al. (2008). High incidence of squeaking in THAs with alumina ceramic-onceramic bearings. Clin Orthop Relat Res, 466, 2008: 1438-1443

Kim YH. (2005). Comparison of polyethylene wear associated with cobalt-chromium and zirconia heads after total hip replacement. A prospective, randomized study. J Bone Joint Surg Am, 87(8), 2005: 1769-1776

Komistek RD, et al. (2002). In vivo comparison of hip separation after metal-on-metal or metal-on-polyethylene total hip arthroplasty. J Bone Joint Surg Am, 84, 2002: 18361841

Kwon YM, et al. (2009). Dose-dependent cytotoxicity of clinically relevant cobalt nanoparticles and ions on macrophages in vitro. Biomed Mater, 4, 2009: 18-25

Lerouge S, et al. (1997). Ceramic-ceramic and metal-polyethylene total hip replacements comparison of pseudomembranes after loosening. J Bone Joint Surg Br, 79B, 1997: 135 
Lombardi AV, et al. (2010). Delta Ceramic-on-Alumina Ceramic Articulation in Primary THA; Prospective, Randomized FDA-IDE Study and Retrieval Analysis. Clin Orthop Relat Res, 468, 2010: 367-374

Lundberga JH, et al. (2006). Problematic sites of third body embedment in polyethylene for total hip wear acceleration. Journal of Biomechanics, 39, 2006: 1208-1212

MacDonald SJ. (2004). Can a safe level for metal ions in patients with metal-on-metal total hip arthroplasties be determined? J Arthroplasty, 19 (Suppl 3), 2004: 71-77

Malviya A et al. (2010). Metal-on-Metal total hip arthroplasty. J Bone Joint Surg Am, 92, 2010: $1675-1683$

McKellop H, et al. (1999). Development of an extremely wear-resistant ultrahigh molecular weight polyethylene for total hip replacements. J Orthop Res, 17, 1999: 157-167

Mehmood S, et al. (2008). Review of ceramic-on-ceramic total hip arthroplasty. J Surg Ortho Adv, 17, 2008: 45-50

Min BW, et al. (2007). The Delayed Fracture of a Ceramic Insert With Modern Ceramic Total Hip Replacement. J Arthroplasty, 22(1), 2007: 136-139

Moore KD, et al. (2008). Early failure of a cross-linked polyethylene acetabular liner. A case report. J Bone Joint Surg Am, 90, 2008: 2499-2504

Muratoglu OK, et al. (2001). A novel method of cross-linking ultra-high-molecular-weight polyethylene to improve wear, reduce oxidation, and retain mechanical properties. J Arthroplasty, 16, 2001: 149

Nam KW, et al. (2007). Alumina-Debris-Induced Osteolysis in Contemporary Alumina-onAlumina Total Hip Arthroplasty A Case Report. J Bone Joint Surg Am, 89, 2007: 2499-2503

Older J. (2002). Charnley low-friction arthroplasty: a worldwide retrospective review at 15 to 20 years. J Arthroplasty, 17, 2002: 675-680

Park YS, et al. (2006). Ceramic failure after total hip arthroplasty with an alumina-onalumina bearing. J Bone Joint Surg Am, 88, 2006: 780-787

Poggie RA, et al. (2007). Failure analysis of a ceramic bearing acetabular component. J Bone Joint Surg Am, 89, 2007: 367-375

Raimondi MT, et al. (2001). Quantitative evaluation of the prosthetic head damage induced by microscopic third-body particles in total hip replacement. J Biomed Mater Res, 58(4), 2001: 436-448

Ranawat AS, Ranawat CS. (2007). The squeaking hip: a cause for concern-agrees. Orthopedics, 30, 2007: 738-743

Restrepo C,et al. (2008). The noisy ceramic hip: is component malpositioning the cause? J Arthroplasty, 23, 2008: 643-649

Rieker CB, et al. (2005). Influence of the clearance on in-vitro tribology of large diameter metal-on-metal articulations pertaining to resurfacing hip implants. Orthop Clin North Am, 36, 2005: 135-142

Rodrı'guez JA, et al. (2008). Squeaking in total hip replacement: a cause for concern. Orthopedics, 31, 2008: 877-878

Ro“hrl SM, et al. (2007). Very low wear of non-remelted highly-linked polyethylene cups. An RSA study lasting up to 6 years. Acta Orthop, 78(6), 2007: 739-745 
Savarino L, et al. (2006). Differences in ion release after ceramic-on-ceramic and metal-onmetal total hip replacement. Medium-term follow-up. J Bone Joint Surg Br, 88, 2006: $472-476$

Scholes SC, Unsworth A. (2000). Comparison of friction and lubrication of different hip prostheses. Proc Inst Mech Eng, 214, 2000: 49-57

Scholes SC, et al. (2006b). Compliant layer acetabular cups: Friction testing of a range of materials and designs for a new generation of prosthesis that mimics the natural joint. Proc Inst Mech Eng, 220, 2006: 583-596

Skipor AK, et al. (2002). Serum and urine metal levels in patients with metal-on-metal surface arthroplasty. J Mater Sci Mater Med, 13, 2002:1227-1234

Smith SL, et al. (2001a). The effect of femoral head diameter upon lubrication and wear of metal-on-metal total hip replacements. Proc Inst Mech Eng, 215, 2001: 161-170

Smith SL, et al. (2001b). The lubrication of metal-on-metal total hip joints: a slide down the Stribeck curve. Proc Inst Mech Eng, 215, 2001: 483-493

Stewart TD, et al. (2001). Long-term wear of HIPed alumina on alumina bearings for THR under microseparation conditions. J Mater Sci, 12, 2001: 1053-1056

Stewart TD, et al. (2003). Long-term wear of ceramic matrix composite materials for hip prostheses under severe swing phase microseparation. J Biomed Mater Res, 66, 2003 : 567-573

Tateiwa T, et al. (2008). Ceramic total hip arthroplasty in the United States: safety and risk issues revisited. Am J Orthop, 37, 2008: 26-31

Taylor S, et al. (2007). The role of stripe wear causing acoustic emissions from alumina ceramic-on-ceramic bearings. J Arthroplasty, 22(7 Suppl. 3), 2007: 47-51

Teoh SH, et al. (2002). An elasto-plastic finite element model for polyethylene wear in total hip arthroplasty. Biomechanics, 35, 2002: 323-330

Tharani R, et al. (2001). The risk of cancer following total hip or knee arthroplasty. J Bone Joint Surg Am, 83, 2001: 774-780

Udofia IJ, Jin ZM. (2003). Elastohydrodynamic lubrication analysis of metal-on-metal hip resurfacing prostheses. J. Biomech, 36, 2003: 537-544

Urban RM, et al. (2000). Dissemination of wear particles to liver, spleen, and abdominal lymph nodes of patients with hip or knee replacement. J Bone Joint Surg Am, 82, 2000: 457-477

Urban JA, et al. (2001). Ceramic-on-polyethylene bearing surfaces in total hip arthroplasty: seventeen to twenty-one year results. J Bone Joint Surg Am, 83, 2001: 1688-1694

Walter WL, et al. (2004). Edge loading in third generation alumina ceramic-on-ceramic bearings: stripe wear. J Arthroplasty, 19, 2004: 402-413

Walter WL, et al. (2007). Squeaking in ceramic-on-ceramic hips: the importance of acetabular component orientation. J Arthroplasty, 22, 2007: 496-503

Walter WL, et al. (2008). Squeaking hips. J Bone Joint Surg Am, 90 (Suppl 4), 2008: 102-111

Wang A, et al. (1999) Suitability and limitations of carbon fiber reinforced PEEK composite as bearing surfaces for total joint replacement. Wear, 229, 1999: 724-727

Whittingham-Jones PM, et al. (2008). Immune Responses in Patients with Metal-on-Metal Hip Articulations: A Long-term Follow-up. J Arthroplasty, 23(8), 2008: 1212-1218 
Williams S, et al. (2004). Comparative wear under different conditions of surface-engineered metal-on-metal bearings for total hip arthroplasty. J Arthroplasty, 19 (Suppl 3), 2004: 112-117

Wimmer MA, et al. (2003). Tribochemical reaction on metal-on-metal hip joint bearings: a comparison between in-vitro and in-vivo results. Wear, 255, 2003: 1007-1024

Yoshitomi H, et al. (2009). Manufacturers Affect Clinical Results of THA with Zirconia Heads A Systematic Review. Clin Orthop Relat Res, 467, 2009: 2349-2355

Ziaee H, et al. (2007). Transplacental transfer of cobalt and chromium in patients with metalon-metal hip arthroplasty: a controlled study. J Bone Joint Surg Br, 89, 2007: 301-305 


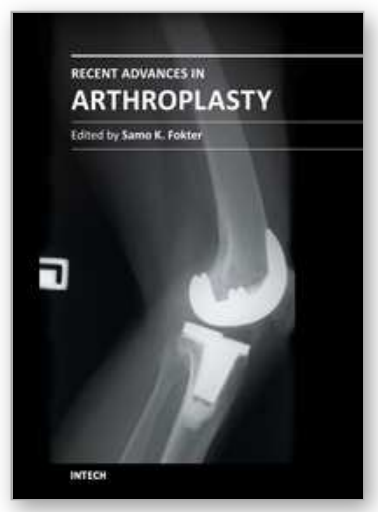

\author{
Recent Advances in Arthroplasty \\ Edited by Dr. Samo Fokter
}

ISBN 978-953-307-990-5

Hard cover, 614 pages

Publisher InTech

Published online 27, January, 2012

Published in print edition January, 2012

The purpose of this book was to offer an overview of recent insights into the current state of arthroplasty. The tremendous long term success of Sir Charnley's total hip arthroplasty has encouraged many researchers to treat pain, improve function and create solutions for higher quality of life. Indeed and as described in a special chapter of this book, arthroplasty is an emerging field in the joints of upper extremity and spine. However, there are inborn complications in any foreign design brought to the human body. First, in the chapter on infections we endeavor to provide a comprehensive, up-to-date analysis and description of the management of this difficult problem. Second, the immune system is faced with a strange material coming in huge amounts of micro-particles from the tribology code. Therefore, great attention to the problem of aseptic loosening has been addressed in special chapters on loosening and on materials currently available for arthroplasty.

\title{
How to reference
}

In order to correctly reference this scholarly work, feel free to copy and paste the following:

Hamid Reza Seyyed Hosseinzadeh, Alireza Eajazi and Ali Sina Shahi (2012). The Bearing Surfaces in Total Hip Arthroplasty - Options, Material Characteristics and Selection, Recent Advances in Arthroplasty, Dr. Samo Fokter (Ed.), ISBN: 978-953-307-990-5, InTech, Available from: http://www.intechopen.com/books/recentadvances-in-arthroplasty/the-bearing-surfaces-in-total-hip-arthroplasty-options-material-characteristics-andselection

\section{INTECH}

open science | open minds

\section{InTech Europe}

University Campus STeP Ri

Slavka Krautzeka 83/A

51000 Rijeka, Croatia

Phone: +385 (51) 770447

Fax: +385 (51) 686166

www.intechopen.com

\section{InTech China}

Unit 405, Office Block, Hotel Equatorial Shanghai

No.65, Yan An Road (West), Shanghai, 200040, China

中国上海市延安西路65号上海国际贵都大饭店办公楼 405 单元

Phone: +86-21-62489820

Fax: $+86-21-62489821$ 
(C) 2012 The Author(s). Licensee IntechOpen. This is an open access article distributed under the terms of the Creative Commons Attribution 3.0 License, which permits unrestricted use, distribution, and reproduction in any medium, provided the original work is properly cited. 